JOURNAL OF THE AMERICAN MATHEMATICAL SOCIETY

Volume 9, Number 1, January 1996

\title{
THE GROTHENDIECK DUALITY THEOREM VIA BOUSFIELD'S TECHNIQUES AND BROWN REPRESENTABILITY
}

\author{
AMNON NEEMAN
}

\section{INTRODUCTION}

Let $f: X \rightarrow Y$ be a proper morphism of schemes. Then, under mild hypotheses on $f, X$, and $Y$, Grothendieck proved that there is a natural isomorphism

$$
R f_{*} \mathcal{R} \operatorname{Hom}_{X}\left(x, f^{!} y\right) \simeq \mathcal{R H o m}{ }_{Y}\left(R f_{*} x, y\right)
$$

of objects in the derived category. We should perhaps briefly remind the reader what this means.

Let $D^{+}(q c / X)$ be the derived category of bounded-below chain complexes of quasi-coherent sheaves on $X$. Let $R f_{*}: D^{+}(q c / X) \rightarrow D^{+}(q c / Y)$ be the right derived functor of $f_{*}$. Then the statement above asserts that $R f_{*}$ has a right adjoint, denoted $f^{!}$, and that $f^{!}$behaves well with respect to pullbacks by open immersions.

Grothendieck's original proof of the existence of $f^{!}$was constructive. It amounted to a local computation. Since derived categories are basically unsuited for local computations, the argument turns out to be quite unpleasant; see [8].

There is an abstract way to prove the existence of $f^{!}$in the literature. It is due to Deligne [7]. The approach was developed and elaborated by Verdier [17], where it is shown that almost everything in [8] can be obtained directly from Deligne's result.

All these results assumed the scheme $X$ Noetherian. Lipman recently developed a deep theory for removing the Noetherian hypotheses. The reader is referred to [10] and [11].

Unfortunately, none of the approaches generalizes well to $D$-modules. Given a morphism $f: X \rightarrow Y$, one can define a morphism $R f_{+}$on the corresponding derived categories of complexes of $D$-modules. It turns out that $R f_{+}$has a right adjoint. But the existence of the right adjoint has until now always been proved by factoring $f$ suitably, defining certain trace maps which $\grave{a}$ priori depend on the factorization, and finally proving that they are independent of factorizations.

What we propose to do here is show that all of the results are direct consequences of a very simple, general statement about triangulated functors on triangulated categories.

Received by the editors January 24, 1994 and, in revised form, December 2, 1994.

1991 Mathematics Subject Classification. Primary 14F05, 55P42.

The author's research was partly supported by NSF grant DMS-9204940.

(C)1996 American Mathematical Society 
Definition 1.7. Let $\mathcal{S}$ be a triangulated category. Suppose that all small coproducts exist in $\mathcal{S}$. Suppose there exists a set $S$ of objects of $\mathcal{S}$ such that

(1) For every $s \in S, \operatorname{Hom}(s,-)$ commutes with coproducts.

(2) If $y$ is an object of $\mathcal{S}$ and $\operatorname{Hom}(s, y)=0$ for all $s \in S$, then $y=0$.

Such a triangulated category $\mathcal{S}$ is called compactly generated.

The Brown Representability Theorem, in one version, states:

Theorem 4.1. Let $\mathcal{S}$ be a compactly generated triangulated category, $\mathcal{T}$ any triangulated category. Let $F: \mathcal{S} \rightarrow \mathcal{T}$ be a triangulated functor. Suppose $F$ respects coproducts; that is, the natural map

$$
\coprod_{\lambda \in \Lambda} F\left(s_{\lambda}\right) \rightarrow F\left(\coprod_{\lambda \in \Lambda} s_{\lambda}\right)
$$

is an isomorphism. Note that although we are not assuming that $\mathcal{T}$ has coproducts, we are assuming that the object on the right is a coproduct of the objects on the left. Then there exists a right adjoint for $F$, namely a functor $G: \mathcal{T} \rightarrow \mathcal{S}$, for which there is a natural isomorphism

$$
\operatorname{Hom}_{\mathcal{S}}(x, G y)=\operatorname{Hom}_{\mathcal{T}}(F x, y) .
$$

We will see that Grothendieck duality is an immediate consequence. Even the non-noetherian statements come very cheaply.

Sections 1 and 2 are introductory. They give the definitions and elementary properties of compactly generated categories. They also discuss why the categories one naturally wants to consider are examples; in particular, the derived category of quasi-coherent sheaves on $X$, and the derived category of quasi-coherent sheaves of $D$-modules. There is, however, a technical point. In the context of Brown representability, it is essential to have triangulated categories with direct sums. Thus we must work with unbounded derived categories. Sections 1 and 2 also discuss why the natural functors one might consider, for instance $R f_{*}$, respect coproducts.

It should be definitely be noted that nothing in Section 1 is new. Except for the terminology, the results can certainly all be found in SGA6. There is also an excellent exposition of them in Thomason's [16]. But there are two reasons for giving a complete and self-contained exposition of these facts. One is to keep this article self-contained. But more importantly, both [1] and [16] are pre-Bousfield. This needs to be made precise. As the reader will easily observe by studying the dates, [16] came long after Bousfield's [3], [4] and [5]. But as far as the author knows, it was not until [2] that it was realised that Bousfield's techniques applied to these problems. And [2] is more recent than [16]. Ever since [2], the present author has delighted in taking every opportunity to point out that Bousfield's techniques make all the old results much clearer, more general and easier to prove. Section 1 is to be taken in this vein. In fact, perhaps the entire article is little more than a manifestation of the above.

As for Section 2, it redoes the classical theory from the new perspective offered by Thomason's localisation theorem. Classically, the proof that $D(q c / X)$ is compactly generated relied on finding enough line bundles on $X$. It follows from Thomason's localisation theorem that one does not need line bundles. This is explained in Section 2. Again, the observation is not new, it was first made by Thomason in 
[16]. Section 2 is the only section in this article which assumes familiarity either with Thomason's article on the subject, or with [13]. There is, for the reader's benefit, a summary of the needed results in Theorem 2.1. A reader willing to assume that all his/her schemes have ample line bundles can skip Section 2, except for the last page and a half which discuss homotopy colimits.

There is a technical point which perhaps deserves mention. In the literature, one frequently considers some other triangulated categories. For instance, the category $D(q c / X)$ of complexes of quasi-coherent sheaves on $X$ may be replaced by $D_{q c}(X)$, the category of complexes of sheaves of $\mathcal{O}_{X}$ modules on $X$, with quasi-coherent cohomology. There is a natural functor

$$
F: D(q c / X) \longrightarrow D_{q c}(X) \text {. }
$$

It turns out that this functor is an equivalence of categories if $X$ is quasi-compact and separated; see [2], Corollary 5.5. A similar statement holds for the derived categories of $D$-modules; I leave it to the reader to state and prove the analogue. We will make no explicit use of this fact. It is only mentioned to comfort the experts who might be concerned about such things.

A similar statement also is valid for maps. Given a morphism $f: X \rightarrow Y$, there are induced maps $R f_{*}: D(q c / X) \rightarrow D(q c / Y)$ and $R f_{*}: D(X) \rightarrow D(Y)$. It is comforting to know that they agree; there is a commutative diagram

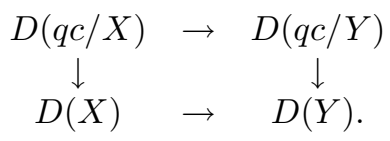

It is possible to give a proof based on homotopy theoretic ideas as in [2], but for a written proof we refer the reader to [10], Proposition 3.9.2.

Sections 3 and 4 contain the proof of the Brown Representability Theorem, Theorem 4.1. The proof itself is very short and simple, and although not very different from Brown's proof in [4], we include it for the reader's convenience.

The last two sections, Sections 5 and 6 , treat the same problems that are addressed by Verdier in [17]. What is different here is (1) that we work with unbounded derived categories, and (2) that the argument is entirely based on the behaviour of coproducts. In Section 5 we ask when does the right adjoint $f^{!}$of $R f_{*}: D(q c / X) \rightarrow D(q c / Y)$ respect coproducts? It turns out that one can give a simple, satisfactory criterion, and what makes the question "natural" is that $f$ ! respects coproducts precisely when

$$
f^{!}(y) \simeq L f^{*}(y) \otimes f^{!} \mathcal{O}_{Y} .
$$

In other words, $f$ ! respects coproducts when there is a dualizing complex $f^{!} \mathcal{O}_{Y}$, and $f^{!}$is given as tensor product with $f^{!} \mathcal{O}_{Y}$. Note that we will always write $\otimes$ for the left derived functor ${ }^{L} \otimes$.

The final question we address is when the functor $f^{!}$localizes well; that is, when it gives an isomorphism in $D(q c / Y)$, as in Grothendieck's original theorem. Although our treatment here is not complete, we give a useful sufficient criterion. The reader should note that Theorem 2 in Verdier [17], p. 394, seems better than the result we get here; but this is partly a delusion. Verdier's theorem is about $D_{q c}^{+}(X)$ whereas Proposition 6.2 deals with $D_{q c}(X)$. The difference between bounded and unbounded derived categories is crucial here. Without boundedness hypotheses Theorem 2 in Verdier [17] fails. Another way to say this is that certain functors that come up in the proof commute with coproducts in $D_{q c}^{+}(X)$ but not with coproducts 
in $D_{q c}(X)$. See the proof of Proposition 6.3 for a proof of Verdier's result based on coproducts (in the non-noetherian case too). See Example 6.5 for a counterexample showing that without some conditions, one cannot expect Verdier's theorem to hold in the unbounded derived category.

It should be emphasised that here also, the topological techniques work without any hypothesis that the schemes be noetherian. We recover, therefore, Lipman's results.

The point of this article is that Grothendieck duality is very easy to prove by homotopy theoretic techniques. It is an immediate consequence of Brown representability. But the reader is not assumed familiar with homotopy theory; hence the first few sections, in which we attempt to give something like a self-contained treatment. The reader is not assumed familiar with much of the literature on the subject, especially not with previous articles by the present author. What is assumed is some familiarity with derived categories. Since we will be working almost entirely with unbounded derived categories, the reader should be familiar with the work of Spaltenstein [15] on extending $R f_{*}$ and tensor products to unbounded complexes. There is a brief account of Spaltenstein's results in Sections 1 and 2 of [2]; this account is recommended because it uses the notations and terminology of the topologists, which we will also follow here.

Finally, I wish to thank Morava for directing me to the problem. I read Residues and Duality many years ago, before I learned any topology. But Morava kept suggesting that there is more there than meets the eye. There is a topological analogue one would like to understand. On rereading Residues and Duality, it became clear to me that even the algebraic geometry could be understood better. I also want to thank Alonso, Jeremias, Kuhn and Lipman for helpful conversations and useful comments. Lipman was especially helpful, reading earlier versions of this manuscript, pointing out gaps and making many suggestions. The referee also pointed out helpful simplifications and corrections.

The $D$-module problem of directly constructing the right adjoint of $R f_{+}$was posed by Nick Katz nine years ago. By a happy accident, I am now able to solve it.

\section{Preliminaries, approached Classically}

Let $\mathcal{T}$ be a triangulated category. There are several hypotheses one likes to place on $\mathcal{T}$ in order to work with it.

Definition 1.1. The category $\mathcal{T}$ is said to contain small coproducts if, for any small set $\Lambda$ and any collection $\left\{t_{\lambda}, \lambda \in \Lambda\right\}$ of objects $t_{\lambda} \in \operatorname{Ob}(\mathcal{T})$ indexed by $\Lambda$, the categorical coproduct

$$
\coprod_{\lambda \in \Lambda} t_{\lambda}
$$

exists in $\mathcal{T}$.

Remark 1.2. It turns out that one can prove two things:

1.2.1. The suspension functor commutes with coproducts; that is the natural map

is an isomorphism.

$$
\coprod_{\lambda \in \Lambda} \Sigma t_{\lambda} \rightarrow \Sigma\left\{\coprod_{\lambda \in \Lambda} t_{\lambda}\right\}
$$


1.2.2. The coproduct of any set of triangles is a triangle.

The proof of this may be found in the yet-to-be-completed [14]. If the reader is unhappy with this, just modify Definition 1.1 so that a category $\mathcal{T}$ containing small coproducts is assumed to satisfy 1.2.1 and 1.2.2.

Example 1.3. Let $X$ be a scheme. Let $D(q c / X)$ be the derived category of chain complexes of quasi-coherent sheaves over $X$.

Suppose $\left\{x_{\lambda} \mid \lambda \in \Lambda\right\}$ is a set of objects of $D(q c / X)$. That is, each $x_{\lambda}$ is a chain complex

$$
\rightarrow x_{\lambda}^{n-1} \stackrel{\partial}{\rightarrow} x_{\lambda}^{n} \stackrel{\partial}{\rightarrow} x_{\lambda}^{n+1} \rightarrow
$$

Then it is nearly trivial that the chain complex

$$
\longrightarrow \coprod_{\lambda \in \Lambda} x_{\lambda}^{n-1} \stackrel{\coprod^{\partial}}{\longrightarrow} \coprod_{\lambda \in \Lambda} x_{\lambda}^{n} \stackrel{\coprod^{\partial}}{\longrightarrow} \coprod_{\lambda \in \Lambda} x_{\lambda}^{n+1} \longrightarrow
$$

is the coproduct of the $x_{\lambda}$ in the category $D(q c / X)$. It is also nearly trivial that coproducts of triangles are triangles. For a proof, see [2], Corollary 1.7.

Lemma 1.4. Let $X$ be a quasi-compact, separated scheme and let $Y$ be a scheme. Let $f: X \rightarrow Y$ be a separated morphism. Let $R f_{*}: D(q c / X) \rightarrow D(q c / Y)$ be the direct image functor. Then the natural map

$$
\coprod_{\lambda \in \Lambda} R f_{*} x_{\lambda} \rightarrow R f_{*}\left\{\coprod_{\lambda \in \Lambda} x_{\lambda}\right\}
$$

is an isomorphism; that is, $R f_{*}$ respects coproducts.

Proof. The question being local in $Y$, we may assume $Y$ affine. Since $X$ is quasicompact, it may be covered by finitely many open affines: $X=\bigcup_{i=1}^{n} U_{i}$, with $U_{i}$ affine. We will prove the lemma by induction on the number $n$ of open affines.

If $n=1$, then $X=U_{1}$ is affine. Thus the map

$$
\operatorname{Spec}(S)=X \rightarrow Y=\operatorname{Spec}(R)
$$

corresponds to a ring homomorphism $R \rightarrow S$. The category $D(q c / X)$ is just $D(S)$, the derived category of chain complexes of $S$-modules. The functor $R f_{*}: D(S) \rightarrow$ $D(R)$ just takes a chain complex of $S$-modules and views it as a chain complex of $R$-modules. This clearly preserves coproducts.

$$
\text { If } n>1 \text {, let } U=U_{1}, V=\bigcup_{i=2}^{n} U_{i} \text {. Then } U \cap V=\bigcup_{i=2}^{n}\left(U_{1} \cap U_{i}\right) \text {, and } U_{1} \cap U_{i} \text { is }
$$

affine because $X$ is separated. Thus both $V$ and $U \cap V$ are unions of $n-1$ affines. By induction, the theorem holds for the maps $f_{U}: U \rightarrow Y, f_{V}: V \rightarrow Y$ and $f_{U \cap V}: U \cap V \rightarrow Y$.

Let $i_{U}: U \hookrightarrow X, i_{V}: V \hookrightarrow X$ and $i_{U \cap V}: U \cap V \hookrightarrow X$ be the open immersions. Then any object $x$ of $D(q c / X)$ admits a triangle: 
(1)

$$
R\left(i_{U \cap V}\right)_{*} i_{U \cap V}^{*}(x)
$$

Thus we deduce a triangle:

$$
\begin{aligned}
& R f_{*}(x) \quad \longrightarrow \quad R f_{*} R\left(i_{U}\right)_{*} i_{U}^{*}(x) \oplus R f_{*} R\left(i_{V}\right)_{*} i_{V}^{*}(x) \\
& \text { (1) } \\
& R f_{*} R\left(i_{U \cap V}\right)_{*} i_{U \cap V}^{*}(x)
\end{aligned}
$$

But now

$$
\begin{aligned}
R f_{*} R\left(i_{U}\right)_{*} & =R\left(f_{U}\right)_{*}, \\
R f_{*} R\left(i_{V}\right)_{*} & =R\left(f_{V}\right)_{*}, \\
R f_{*} R\left(i_{U \cap V}\right) & =R\left(f_{U \cap V}\right)_{*}
\end{aligned}
$$

all commute with coproducts by the induction hypothesis. The functors $i_{U}^{*}, i_{V}^{*}$ and $i_{U \cap V}^{*}$ commute with coproducts because they have right adjoints. Therefore, in the morphism of triangles

$$
\begin{aligned}
& \coprod_{\lambda \in \Lambda} R f_{*}\left(x_{\lambda}\right) \rightarrow \coprod_{\lambda \in \Lambda}\left[R\left(f_{U}\right)_{*} i_{U}^{*}\left(x_{\lambda}\right) \oplus R\left(f_{V}\right)_{*} i_{V}^{*}\left(x_{\lambda}\right)\right] \rightarrow \coprod_{\lambda \in \Lambda} R\left(f_{U \cap V}\right)_{*} i_{U \cap V}^{*}\left(x_{\lambda}\right) \\
& R f_{*}\left(\coprod_{\lambda \in \Lambda}^{\alpha \downarrow} x_{\lambda}\right) \rightarrow R\left(f_{U}\right)_{*} i_{U}^{*}\left(\coprod_{\lambda \in \Lambda} x_{\lambda}\right)^{\beta \downarrow} \oplus R\left(f_{V}\right)_{*} i_{V}^{*}\left(\coprod_{\lambda \in \Lambda} x_{\lambda}\right) \rightarrow R\left(f_{U \cap V}\right)_{*} i_{U \cap V}^{*}\left(\coprod_{\lambda \in \Lambda} x_{\lambda}\right)
\end{aligned}
$$

the maps $\beta$ and $\gamma$ are isomorphisms; hence so is $\alpha$.

Corollary 1.5. If in Lemma 1.4 we take $Y$ to be the scheme $\operatorname{Spec}(\mathbb{Z})$, then $R f_{*}$ is just the derived functor of the global section functor. We deduce that if $X$ is a quasi-compact, separated scheme, then the functors $H^{i}$

$$
H^{i}(X,-): D(q c / X) \rightarrow\{\text { abelian groups }\}
$$

respect coproducts.

Definition 1.6. An object $c$ of $\mathcal{T}$ is called compact if, for any coproduct of objects of $\mathcal{T}$,

$$
\operatorname{Hom}_{\mathcal{T}}\left(c, \coprod_{\lambda \in \Lambda} t_{\lambda}\right)=\coprod_{\lambda \in \Lambda} \operatorname{Hom}_{\mathcal{T}}\left(c, t_{\lambda}\right) .
$$

Observation. The suspension of a compact object is compact. 
Definition 1.7. The triangulated category $\mathcal{T}$ is called compactly generated if $\mathcal{T}$ contains small coproducts, and there exists a small set $T$ of compact objects of $\mathcal{T}$, such that

$$
\operatorname{Hom}(T, x)=0 \Rightarrow x=0
$$

In other words, if $x$ is an object of $\mathcal{T}$, and for every $t \in T$, $\operatorname{Hom}(t, x)=0$, then $x$ must be the zero object.

Definition 1.8. If $\mathcal{T}$ is a compactly generated triangulated category, then a set $T$ of compact objects of $\mathcal{T}$ is called a generating set if

(1) $\operatorname{Hom}(T, x)=0 \Rightarrow x=0$;

(2) $T$ is closed under suspension; $T=\Sigma T$.

Remark 1.9. Let $T$ be any set of objects in $\mathcal{T}$ as in Definition 1.7. Then

$$
\bigcup_{i \in \mathbb{Z}} \Sigma^{i} T
$$

is a generating set as in Definition 1.8. (1) holds because it holds for $T$; and clearly the set

$$
\bigcup_{i \in \mathbb{Z}} \Sigma^{i} T
$$

is stable under suspension. It needs only be remarked that any suspension of a compact object is compact, and hence the set consists only of compact objects of $\mathcal{T}$.

Example 1.10. Let $X$ be a quasi-compact, separated scheme. Let $\mathcal{T}=D(q c / X)$ be the category of chain complexes of quasi-coherent sheaves on $X$. By Example 1.3 we know that $\mathcal{T}$ contains small coproducts. Now let $\mathcal{L}$ be any line bundle on $X$. View $\mathcal{L}$ as an object of $D(q c / X)$; it is the complex of sheaves which is just $\mathcal{L}$ in degree 0 . Then

$$
\operatorname{Hom}\left(\mathcal{L}, \coprod_{\lambda \in \Lambda} t_{\lambda}\right)=H^{0}\left(\mathcal{L}^{-1} \otimes \coprod_{\lambda \in \Lambda} t_{\lambda}\right) .
$$

Now tensor product respect coproducts, since it has a right adjoint. The functor $H^{0}$ respects coproducts by Corollary 1.5. It follows that $\mathcal{L}$ is a compact object of $\mathcal{T}=D(q c / X)$.

Now suppose $\mathcal{L}$ is an ample line bundle. For any $m \in \mathbb{Z}, \mathcal{L}^{m}$ is compact. For any $n \in \mathbb{Z}, \Sigma^{n} \mathcal{L}^{m}$ is also compact. Let

$$
T=\left\{\Sigma^{n} \mathcal{L}^{m} \mid m, n \in \mathbb{Z}\right\} .
$$

I assert that $T$ is a generating set for $\mathcal{T}$. Suppose $x \neq 0$ is an object of $\mathcal{T}$. Then it has some non-trivial sheaf cohomology; for some $n, \mathcal{H}^{-n}(x) \neq 0$. But $\mathcal{H}^{-n}(x)$ is a quasi-coherent sheaf on $X$, and because $\mathcal{L}$ is ample, $\mathcal{L}^{t} \otimes \mathcal{H}^{-n}(x)$ has non-zero global sections for some $t>>0$. If $x$ is the complex

$$
\rightarrow x^{-n-1} \stackrel{\partial}{\rightarrow} x^{-n} \stackrel{\partial}{\rightarrow} x^{-n+1} \rightarrow,
$$

then there is a surjective map of quasi-coherent sheaves on $X$

$$
\operatorname{ker}\left(x^{-n} \rightarrow x^{-n+1}\right) \rightarrow \mathcal{H}^{-n}(x),
$$

and it follows that, choosing $t$ large enough, we can find a class

$$
s \in H^{0}\left(\mathcal{L}^{t} \otimes x^{-n}\right)
$$


which maps to 0 in $H^{0}\left(\mathcal{L}^{t} \otimes x^{-n+1}\right)$, and whose image in $H^{0}\left(\mathcal{H}^{-n}\left(\mathcal{L}^{t} \otimes x\right)\right)$ is non-zero. This immediately gives us a non-zero map

$$
\Sigma^{n} \mathcal{L}^{-t} \rightarrow x
$$

If all such maps vanish, so must $x$.

Example 1.11. In Example 1.10 we can replace a single ample line bundle by a family; if $X$ admits a family of line bundles $\left\{\mathcal{L}_{\alpha} \mid \alpha \in A\right\}$ which is jointly ample, then

$$
T=\left\{\Sigma^{n} \mathcal{L}_{\alpha}^{m} \mid m, n \in \mathbb{Z}, \alpha \in A\right\}
$$

will do for a generating set of compact objects. See [1], p. 171.

Example 1.12. Let $X$ be a smooth quasi-compact variety of finite type over a field $k$ of characteristic zero. Let $\mathcal{T}=D\left(\frac{q c D \text {-modules }}{X}\right)$ be the derived category of chain complexes of quasi-coherent $D$-modules over $X$. Once again, it is trivial that $\mathcal{T}$ contains small coproducts.

Because $X$ is smooth, one knows by a trick of Kleiman that there is an ample family of line bundles; cover $X$ by open affines $X=\bigcup_{i \in I} U_{i}$ and let $\mathcal{L}_{i}$ be the line bundle $\mathcal{O}\left(D_{i}\right)$, where $D_{i}$ is the divisor $X-U_{i}$. Then the $\mathcal{L}_{i}$ 's form an ample family.

Now observe

$$
\operatorname{Hom}_{D\left(\frac{q c D \text {-modules }}{X}\right)}\left(\mathcal{D}_{X} \otimes_{\mathcal{O}_{X}} \mathcal{L}_{i}^{m}, x\right)=\operatorname{Hom}_{D(q c / X)}\left(\mathcal{L}_{i}^{m}, x\right) .
$$

From this it follows that $\Sigma^{n}\left(\mathcal{D}_{X} \otimes_{\mathcal{O}_{X}} \mathcal{L}_{i}^{m}\right)$ are compact for all $i, m$ and $n$, and the set

$$
T=\left\{\Sigma^{n}\left(\mathcal{D}_{X} \otimes_{\mathcal{O}_{X}} \mathcal{L}_{i}^{m}\right) \mid i \in I, \quad m, n \in \mathbb{Z}\right\}
$$

is a generating set.

Example 1.13. Let $X$ be a quasi-compact, separated scheme. In Example 1.10 we proved that any line bundle $\mathcal{L}$ on $X$, viewed as an object of $D(q c / X)$, is compact. Given ample families of line bundles on $X$, we used this to construct a generating set.

Let us now observe that if $c \in D(q c / X)$ is any perfect complex on $X$, it is compact. Recall that a complex $c$ is perfect if, locally on $X$, it is isomorphic to a bounded complex of finitely generated, projective $\mathcal{O}_{X}$-modules.

Proof. Let $\coprod_{\lambda \in \Lambda} x_{\lambda}$ be a coproduct in $D(q c / X)$. Let

$$
\mathcal{R H o m}\left(c, \coprod_{\lambda \in \Lambda} x_{\lambda}\right)
$$

be the sheaf $\mathcal{R} H o m$; it is an object of $D(q c / X)$. I assert that the natural map in $D(q c / X)$,

$$
\phi_{c}: \coprod_{\lambda \in \Lambda} \mathcal{R} H o m\left(c, x_{\lambda}\right) \rightarrow \mathcal{R H o m}\left(c, \coprod_{\lambda \in \Lambda} x_{\lambda}\right)
$$


is an isomorphism whenever $c$ is a perfect complex. The problem is local in $X$; we may therefore assume that $X$ is affine, and $c$ is a bounded complex of finitely generated projective $\mathcal{O}_{X}$-modules.

If $c$ is $\Sigma^{m} \mathcal{O}_{X}$, then $\phi_{c}$ is clearly an isomorphism. If $c$ is $\Sigma^{m} \mathcal{O}_{X}^{n}$, a finite direct sum of $\Sigma^{m} \mathcal{O}_{X}$ 's, then $\phi_{c}$ is also an isomorphism. If $c=c^{\prime} \oplus c^{\prime \prime}$ and $\phi_{c}$ is an isomorphism, so are $\phi_{c^{\prime}}$ and $\phi_{c^{\prime \prime}}$. Hence $\phi_{c}$ is an isomorphism whenever $c$ is a suspension of a finitely generated projective module.

Now if we have a triangle

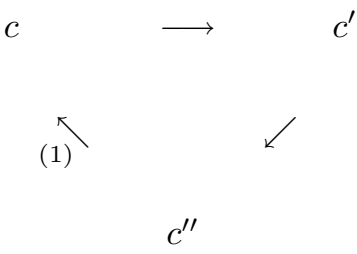

and $\phi_{\Sigma^{m} c^{\prime}}$ and $\phi_{\Sigma^{m} c^{\prime \prime}}$ are isomorphisms for all $m \in \mathbb{Z}$, then it follows from the 5lemma that $\phi_{\Sigma^{m} c}$ is an isomorphism for all $m \in \mathbb{Z}$. Therefore the full subcategory of $c$ 's such that $\phi_{\Sigma^{m} c}$ is an isomorphism for all $m \in \mathbb{Z}$ is triangulated and contains the finitely generated projective $\mathcal{O}_{X}$-modules. Hence it contains finite complexes of finitely generated projectives.

Thus we have proved that for $c$ a perfect complex,

$$
\phi_{c}: \coprod_{\lambda \in \Lambda} \mathcal{R} H o m\left(c, x_{\lambda}\right) \rightarrow \mathcal{R} H o m\left(c, \coprod_{\lambda \in \Lambda} x_{\lambda}\right)
$$

is an isomorphism. But

$$
\begin{aligned}
\operatorname{Hom}\left(c, \coprod_{\lambda \in \Lambda} x_{\lambda}\right) & =H^{0}\left[\mathcal{R} \operatorname{Hom}\left(c, \coprod_{\lambda \in \Lambda} x_{\lambda}\right)\right] \\
& =H^{0}\left[\coprod_{\lambda \in \Lambda} \mathcal{R} \operatorname{Hom}\left(c, x_{\lambda}\right)\right] \\
& =\coprod_{\lambda \in \Lambda} H^{0}\left[\mathcal{R} \operatorname{Hom}\left(c, x_{\lambda}\right)\right] \\
& =\coprod_{\lambda \in \Lambda} \operatorname{Hom}\left(c, x_{\lambda}\right),
\end{aligned}
$$

where the third equality is by Corollary 1.5, which assures us that $H^{0}$ respects coproducts.

Example 1.14. Let $X$ be a quasi-compact, separated smooth scheme of finite type over a field $k$ of characteristic 0 . Then by Example 1.12 we know that objects of the form $\Sigma^{n}\left\{\mathcal{D}_{X} \otimes_{\mathcal{O}_{X}} \mathcal{L}\right\}$ are compact in $\mathcal{T}=D\left(\frac{q c D \text {-modules }}{X}\right)$. But much as in Example 1.13, it can be shown that any bounded complex with coherent cohomology is compact. Note that because $\mathcal{D}_{X}$ is locally of finite projective dimension, any coherent sheaf can locally be replaced by a finite chain complex of finitely generated projectives. We leave the details to the reader. 


\section{The APPROACH USING ThOMASON'S LOCALISATION THEOREM}

Let $\mathcal{T}$ be a triangulated category. In this section, and for the remainder of the article, we adopt the notation that $\mathcal{T}^{c}$ stands for the full subcategory of compact objects in $\mathcal{T}$.

Let $X$ be a quasi-compact, separated scheme. In Section 1 we saw how to prove that the category $D(q c / X)$ is compactly generated, provided $X$ has an ample family of line bundles; see Example 1.11. On the other hand, we also know that if $X$ is arbitrary (that is, quasi-compact and separated but not necessarily possessing any line bundles), every perfect complex on $X$ is compact. In this section we will use Thomason's localisation theorem to prove that $D(q c / X)$ is compactly generated, even without line bundles. First, we quote the theorem to which we will appeal.

Theorem 2.1. Let $\mathcal{S}$ be a compactly generated triangulated category. Let $R$ be a set of compact objects of $\mathcal{S}$ closed under suspension. Let $\mathcal{R}$ be the smallest full subcategory of $\mathcal{S}$ containing $R$ and closed with respect to coproducts and triangles. Let $\mathcal{T}$ be the Verdier quotient $\mathcal{S} / \mathcal{R}$. Then we know:

2.1.1. The category $\mathcal{R}$ is compactly generated, with $R$ as a generating set.

2.1.2. If $R$ happens to be a generating set for all of $\mathcal{S}$, then $\mathcal{R}=\mathcal{S}$.

2.1.3. If $R \subset \mathcal{R}$ is closed under the formation of triangles and direct summands, then it is all of $\mathcal{R}^{c}$. In any case, $\mathcal{R}^{c}=\mathcal{R} \cap \mathcal{S}^{c}$.

2.1.4. Suppose $t$ is a compact object of $\mathcal{T}$. Then there is an object $t^{\prime} \in \mathcal{T}^{c}$, an object $s \in \mathcal{S}^{c}$ and an isomorphism in $\mathcal{T}$

$$
s \simeq t \oplus t^{\prime}
$$

Thus, $t$ might not be isomorphic in $\mathcal{T}$ to a compact object in $\mathcal{S}$, but it is the direct summand of an object isomorphic in $\mathcal{T}$ to a compact object of $\mathcal{S}$. Furthermore, $t^{\prime}$ may be chosen to be $\Sigma t$, or any other object whose sum with $t$ is zero in $K_{0}$.

2.1.5. Given an object $s \in \mathcal{S}^{c}$, an object $s^{\prime} \in \mathcal{S}$, and a morphism in $\mathcal{T} s \rightarrow s^{\prime}$, then there is a diagram in $\mathcal{S}$

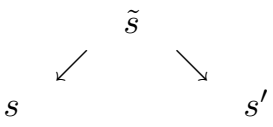

where $\tilde{s}$ is compact, in the triangle $r \rightarrow \tilde{s} \rightarrow s \rightarrow \Sigma r$, the object $r$ is in $\mathcal{R}^{c}$, and when we reduce the diagram to $\mathcal{T}$, the composite of the map $\tilde{s} \rightarrow s^{\prime}$ with the inverse of $\tilde{s} \rightarrow s$ is the given map $s \rightarrow s^{\prime}$.

Remark 2.2. For triangulated categories $D(q c / X)$ where $X$ is a scheme, the theorem is due to Thomason [16]. In the generality in which the theorem is stated, it may be found in [13]. Note that in [13] we assume not only that $\mathcal{S}$ is compactly generated, but that the generating set may be taken to be $\mathcal{S}^{c}$; in particular, we assume $\mathcal{S}^{c}$ to be small. The reader will note that this is inessential to any of the arguments in [13]. The only point where the proof uses the hypothesis on the smallness of $\mathcal{S}^{c}$ is in showing 2.1.4., and that comes at the very end. So in any case we know the other properties. And in the proof of 2.1.4., it suffices to know that $\mathcal{S}$ is compactly generated, and that by 2.1.1. it then follows that if $S$ is a generating set, the category $\mathcal{R} \subset \mathcal{S}$ which is the smallest category containing $S$ and closed with respect to triangles and coproducts is all of $\mathcal{S}$.

With this comment, we now give the references for the proofs. 2.1.2. really follows from the proof in [13]. In the notation there, if $j^{*}: \mathcal{S} \longrightarrow \mathcal{T}$ is the natural 
functor and $j_{*}: \mathcal{T} \longrightarrow \mathcal{S}$ its right adjoint, then the objects $j_{*} j^{*} x$ are characterised by the fact that $\operatorname{Hom}\left(R, j_{*} j^{*} x\right)=0$. Since $R$ generates $\mathcal{S}$, it follows that for every $x, j_{*} j^{*} x=0$. But the identity on $j^{*} x$ factors as

$$
j^{*} x \rightarrow j^{*} j_{*} j^{*} x \rightarrow j^{*} x
$$

and the middle object is zero. Hence $j^{*} x=0$. This is true for every $x$, which means that the category $\mathcal{T}$ is zero, and hence $\mathcal{R}=\mathcal{S}$.

For the remaining statements, 2.1.3. is Lemma 2.2 of [13]. 2.1.4. goes as follows. The existence of $t^{\prime}$ is Lemma 2.6 of [13], but as noted above, the proof needs to be modified slightly to account for the fact that we are only assuming $\mathcal{S}$ compactly generated. The fact that $t^{\prime}$ may be taken to be $\Sigma t$, or even anything else which is isomorphic to $-t$ in $K_{0}$, may be found in the appendix to [13] 2.1.5. is Lemma 2.5 of [13]. This leaves us with 2.1.1. which is trivial, so let us give the proof. Suppose $r$ is an object of $\mathcal{R}$ such that $\operatorname{Hom}(R, r)=0$. Consider the full subcategory ${ }^{\perp} r \subset \mathcal{R}$, defined by

$$
{ }^{{ }^{\perp}}=\left\{x \in \mathcal{R} \mid \operatorname{Hom}\left(\Sigma^{n} x, r\right)=0 \text { for all } n \in \mathbb{Z}\right\} .
$$

Clearly, ${ }^{\perp} r$ is triangulated and closed under coproducts, and contains $R$. Hence it must be all of $\mathcal{R}$, in particular ${ }^{\perp} r$ contains $r \in \mathcal{R}$. Thus $\operatorname{Hom}(r, r)=0$, hence $r=0$.

Corollary 2.3. Let $X$ be a quasi-compact, separated scheme, and suppose we know that $D(q c / X)$ is compactly generated, and that the generating set consists of some perfect complexes. Then the category of all perfect complexes on $X$ is nothing other than $D(q c / X)^{c}$.

Remark 2.4. For now, we only know that $D(q c / X)$ is compactly generated when there is an ample family of line bundles. So for now Corollary 2.3 only applies in that case. But this will change by the end of the section.

Proof of Corollary 2.3. Let $\mathcal{S}=D(q c / X)$, and let $R$ be the set of perfect complexes in Theorem 2.1. By hypothesis, $R$ generates $\mathcal{S}$, and hence by 2.1.2., $\mathcal{R}=\mathcal{S}=$ $D(q c / X)$, and $\mathcal{T}$ is trivial. But $R$ is closed with respect to direct summands and triangles. Closure with respect to triangles is clear. Closure with respect to direct summands asserts that a direct summand of a perfect complex is perfect. This is local, so we may assume $X$ affine. For affine $X$ this is Proposition 3.4 in [2]. By 2.1.3. we therefore know that $R=\mathcal{R}^{c}$; every compact object in $D(q c / X)$ is a perfect complex.

Now we come to the existence of compacts on a general $X$.

Proposition 2.5. Let $X$ be a quasi-compact, separated scheme. Then the category $D(q c / X)$ is compactly generated.

It might be useful to state a lemma that clearly implies Proposition 2.5, and which we will prove.

Lemma 2.6. Let $X$ be a quasi-compact, separated scheme. Let $U \subset X$ be a quasicompact, open subscheme. Let $x$ be an arbitrary object of $D(q c / X)$, and let $u$ be a perfect complex in $D(q c / U)$. Suppose that we are given a map in $D(q c / U)$ of the form $u \rightarrow x$. Then there exists a perfect complex $u^{\prime}$ in $D(q c / U)$ so that the map

$$
u \oplus u^{\prime} \stackrel{\pi_{1}}{\rightarrow} u \rightarrow x
$$


lifts to $D(q c / X)$. There exists a perfect complex $\tilde{u}$ on $X$, restricting to $u \oplus u^{\prime}$ on $U$, and a map $\tilde{u} \rightarrow x$, defined on $X$, which restricts on $U$ to the given map $u \oplus u^{\prime} \stackrel{\pi_{1}}{\rightarrow} u \rightarrow x$.

Proof that Lemma 2.6 implies Proposition 2.5. Take $U$ to be an open affine. Then $U$ admits an ample family of line bundles; the trivial bundle is ample. Thus we already know that $U$ is compactly generated, and that the compact objects are the perfect complexes. If $x$ is any object of $D(q c / X)$ and the restriction of $x$ to $U$ is non-zero, there is a perfect complex $u$ on $U$ and a non-zero map $u \rightarrow x$ on $U$. By Lemma 2.6 this map can be extended to a non-zero map $\tilde{u} \rightarrow x$ on all of $X$, where $\tilde{u}$ is perfect. Thus, unless the restriction of $x$ to every open affine $U \subset X$ is zero, there is a non-zero map from a perfect complex to $x$. But if the restriction of $x$ to every open affine $U \subset X$ vanishes, then $x$ vanishes.

Proof of Lemma 2.6. Suppose first that $X$ is affine. Then $X$ has an ample line bundle; after all, the trivial bundle is ample. We therefore already know that $D(q c / X)$ is compactly generated. Furthermore, the compacts are precisely the perfect complexes. Now let $D_{X-U}(q c / X) \subset D(q c / X)$ be the full subcategory whose objects are complexes supported on $X-U$. That is,

$$
D_{X-U}(q c / X)=\{x \in D(q c / X) \mid \text { the restriction of } x \text { to } U \text { is acyclic }\} \text {. }
$$

Lemma 6.1 of [2] shows that even $D_{X-U}(q c / X)$ is compactly generated, in fact, generated by the suspensions of one compact object in $D(q c / X)$.

In Theorem 2.1 , let $\mathcal{S}$ be the category $D(q c / X)$, and let $R$ be a generating set for $D_{X-U}(q c / X)$. This makes $\mathcal{R}=D_{X-U}(q c / X)$. Now $\mathcal{T}$ is easily identified with $D(q c / U)$. Thomason localisation theorem applies, and we discover first that by 2.1.4. the complex $u \oplus \Sigma u$ may be lifted to a perfect complex $\tilde{u}$ in $D(q c / X)$, and then by 2.1.5. that the map $u \oplus \Sigma u \rightarrow x$ can be lifted to a map $\tilde{u} \rightarrow x$ on all of $X$, possibly after changing the choice of $\tilde{u}$ lifting $u \oplus \Sigma u$.

Suppose next that $X=U \cup W$, where $W$ is affine. We know by the above that the restriction of the map $u \rightarrow x$ can be extended from $U \cap W$ to all of $W$. Precisely, there is a perfect complex $\tilde{u}$ on $W$ and a map $\tilde{u} \rightarrow x$ of complexes on $W$, so that the restriction to $U \cap W$ is isomorphic to the map $u \oplus \Sigma u \rightarrow x$. Thus, if $j_{W}: W \rightarrow X, j_{U}: U \rightarrow X$ and $j_{U \cap W}: U \cap W \rightarrow X$ are the open immersions, we have an isomorphism on $U \cap W$ of $\left\{j_{U \cap W}\right\}^{*}\{u \oplus \Sigma u\}$ with $\left\{j_{U \cap W}\right\}^{*} \tilde{u}$. We are given the maps $\beta$ and $\gamma$, which we can complete to a morphism of triangles of complexes on $U \cup W=X$

$$
\begin{array}{ccccc}
\hat{u} & \rightarrow & \left\{j_{U}\right\}_{*}\{u \oplus \Sigma u\} \oplus\left\{j_{W}\right\}_{*} \tilde{u} & \rightarrow & \left\{j_{U \cap W}\right\}_{*}\left\{j_{U \cap W}\right\}^{*}\{u \oplus \Sigma u\} \\
\alpha \downarrow & & & & \\
x & \rightarrow & \left\{j_{U}\right\}_{*}\left\{j_{U}\right\}^{*} x \oplus\left\{j_{W}\right\}_{*}\left\{j_{W}\right\}^{*} x & \rightarrow & \left\{j_{U \cap W}\right\}_{*}\left\{j_{U \cap W}\right\}^{*} x
\end{array}
$$

and it is easy to check that $\hat{u}$ is perfect and the map $\hat{u} \rightarrow x$, defined on all of $X$, is just a lifting of $u \oplus \Sigma u \rightarrow x$ from $U$ to all of $X$.

Since $X$ is quasi-compact, it can be covered by finitely many open affines, and in finitely many steps of extending from $U$ to $U \cup W$, with $W$ affine, we extend to all of $X$.

Theorem 2.1 can also be used to construct compactly generated categories. The point being that given a compactly generated category $\mathcal{S}$ and a set of compact objects $R$ in it, the categories $\mathcal{R}$ and $\mathcal{T}$ are compactly generated. 
Example 2.7. Let $X$ be a smooth, quasi-compact, separated scheme of finite type over a field $k$ of characteristic 0 . Let $\mathcal{S}=D\left(\frac{q c D \text {-modules }}{X}\right)$ be the derived category of chain complexes of quasi-coherent $D$-modules on $X$.

Let $R \subset \mathcal{S}$ be the set

$$
R=\left\{\begin{array}{l|l}
x \in \operatorname{Ob}(\mathcal{S}) & \begin{array}{l}
\mathcal{H}^{i}(x)=0 \text { for all but finitely many } i, \text { and } \\
\mathcal{H}^{i}(x) \text { is holonomic for all } i
\end{array}
\end{array}\right\}
$$

Because holonomic modules are coherent, it follows from Example 1.14 that $R$ consists of compact objects of $\mathcal{T}$. Let $\mathcal{R}$ be as above. Then $\mathcal{R}$ is a compactly generated triangulated category, with $R$ for a generating set. We will call this $\mathcal{R}$ by the name $D($ holo $/ X)$.

The key tool one uses is the homotopy colimit. Let $\mathcal{T}$ be a triangulated category containing small coproducts. Let

$$
X_{0} \rightarrow X_{1} \rightarrow X_{2} \rightarrow \cdots
$$

be a sequence of objects and morphisms in $\mathcal{T}$. Then hocolim $X_{i}$ is by definition the third edge of the triangle:

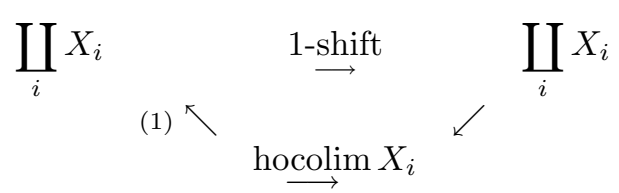

Lemma 2.8. Suppose $c$ is a compact object of $\mathcal{T}$, and suppose

$$
X_{0} \rightarrow X_{1} \rightarrow X_{2} \rightarrow \cdots
$$

is a sequence of objects and morphisms in $\mathcal{T}$. Suppose $\mathcal{T}$ admits small coproducts. Then

$$
\operatorname{Hom}\left(c, \underset{\longrightarrow}{\operatorname{hocolim}} X_{i}\right)=\lim _{\rightarrow} \operatorname{Hom}\left(c, X_{i}\right) .
$$

Proof. Consider the triangle:

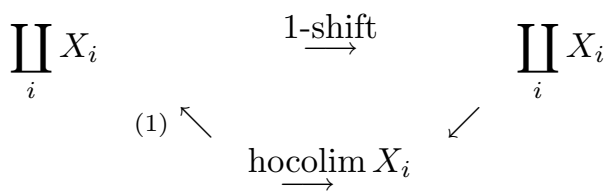

Applying the homological functor $\operatorname{Hom}(c,-)$ we get a long exact sequence. In particular

$$
\begin{aligned}
\operatorname{Hom}\left(c, \coprod_{i} X_{i}\right) \stackrel{\gamma}{\longrightarrow} \operatorname{Hom}\left(c, \operatorname{hocolim} X_{i}\right) \longrightarrow & \operatorname{Hom}\left(c, \coprod_{i} \Sigma X_{i}\right) \\
\downarrow & 1 \text {-shift } \\
& \operatorname{Hom}\left(c, \coprod_{i} \Sigma X_{i}\right)
\end{aligned}
$$


is exact. But $c$ is compact, and hence in the following commutative square the columns are isomorphisms:

$$
\begin{aligned}
& \coprod_{i} \operatorname{Hom}\left(c, \Sigma X_{i}\right) \stackrel{1 \text {-shift }}{\longrightarrow} \coprod_{i} \operatorname{Hom}\left(c, \Sigma X_{i}\right) \\
& |\downarrow 2 \quad| \downarrow 2 \\
& \operatorname{Hom}\left(c, \coprod_{i} \Sigma X_{i}\right) \stackrel{1 \text {-shift }}{\longrightarrow} \operatorname{Hom}\left(c, \coprod_{i} \Sigma X_{i}\right) .
\end{aligned}
$$

But the top row is clearly injective. Hence the bottom row is injective, and we deduce that $\gamma$ is surjective.

We now have a commutative diagram

$$
\begin{aligned}
& \coprod_{i} \operatorname{Hom}\left(c, X_{i}\right) \stackrel{1 \text {-shift }}{\longrightarrow} \coprod_{i} \operatorname{Hom}\left(c, X_{i}\right) \\
& |\downarrow 2 \quad| \downarrow 2 \\
& \operatorname{Hom}\left(c, \coprod_{i} X_{i}\right) \stackrel{1-\text { shift }}{\longrightarrow} \operatorname{Hom}\left(c, \coprod_{i} X_{i}\right) \stackrel{\gamma}{\longrightarrow} \operatorname{Hom}\left(c, \underset{\operatorname{hocolim}}{\longrightarrow} X_{i}\right) \longrightarrow 0
\end{aligned}
$$

where the bottom row is exact. The top row identifies $\operatorname{Hom}\left(c, \underset{\longrightarrow}{\operatorname{hocolim}} X_{i}\right)$ as $\underset{\rightarrow}{\rightarrow}$ $\operatorname{Hom}\left(c, X_{i}\right)$.

\section{BROWN REPRESENTABILITY}

In this section, we will prove:

Theorem 3.1. Let $\mathcal{T}$ be a compactly generated triangulated category. Let $H: \mathcal{T}^{o p} \rightarrow A b$ be a homological functor. That is, $H$ is contravariant and takes triangles to long exact sequences. Suppose the natural map

$$
H\left(\coprod_{\lambda \in \Lambda} t_{\lambda}\right) \rightarrow \prod_{\lambda \in \Lambda} H\left(t_{\lambda}\right)
$$

is an isomorphism for all small coproducts in $\mathcal{T}$. Then $H$ is representable.

Proof. Let $T$ be a generating set for $\mathcal{T}$. Let $U_{0}$ be defined as

$$
U_{0}=\bigcup_{t \in T} H(t) .
$$

Thus elements of $U_{0}$ can be thought of as pairs $(\alpha, t)$ with $\alpha \in H(t)$. Put

$$
X_{0}=\coprod_{(\alpha, t) \in U_{0}} t
$$

Then

$$
H\left(X_{0}\right)=\prod_{(\alpha, t) \in U_{0}} H(t)
$$

and there is an obvious element in $H\left(X_{0}\right)$, namely the element which is $\alpha \in H(t)$ for $(\alpha, t) \in U_{0}$. Call this element $\alpha_{0} \in H\left(X_{0}\right)$. The construction is such that if 
$t \rightarrow X_{0}$ is the inclusion of $t$ into $X_{0}=\coprod_{(\alpha, t) \in U_{0}} t$ corresponding to $(\alpha, t) \in U_{0}$, then the induced map $H\left(X_{0}\right) \rightarrow H(t)$ takes $\alpha_{0} \in H\left(X_{0}\right)$ to $\alpha \in H(t)$.

To give an object $X_{0}$ and an element $\alpha_{0} \in H\left(X_{0}\right)$ is by Yoneda's lemma the same as giving a natural transformation

$$
\phi_{0}: \operatorname{Hom}\left(-, X_{0}\right) \rightarrow H,
$$

and what we have seen is precisely that

$$
\phi_{0}(t): \operatorname{Hom}\left(t, X_{0}\right) \rightarrow H(t)
$$

is surjective, for all $t \in T$.

Suppose that for some $i \geq 0$ we have defined an object $X_{i}$ of $\mathcal{T}$, and a natural transformation

$$
\operatorname{Hom}\left(-, X_{i}\right) \rightarrow H
$$

Define $U_{i+1}$ by

$$
U_{i+1}=\bigcup_{t \in T} \operatorname{ker}\left\{\operatorname{Hom}\left(t, X_{i}\right) \rightarrow H(t)\right\} .
$$

An element of $U_{i+1}$ can be thought of as a pair $(f, t)$ where $t \in T$ and $f: t \rightarrow X_{i}$ is a morphism. Put

$$
K_{i+1}=\coprod_{(f, t) \in U_{i+1}} t
$$

and let $K_{i+1} \rightarrow X_{i}$ be the map which is $f$ on the factor $t$ corresponding to the pair $(f, t)$. Let $X_{i+1}$ be given by the triangle:

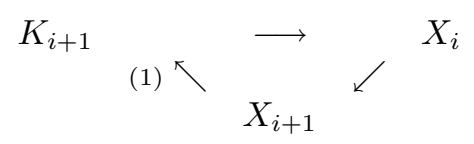

We have a map $\operatorname{Hom}\left(-, X_{i}\right) \rightarrow H$, which by Yoneda's lemma corresponds to an element $\alpha_{i} \in H\left(X_{i}\right)$. Under the map

$$
\begin{aligned}
H\left(X_{i}\right) \rightarrow H\left(K_{i+1}\right) & =H\left(\coprod_{(f, t) \in U_{i+1}} t\right) \\
& =\prod_{(f, t) \in U_{i+1}} H(t)
\end{aligned}
$$

the element $\alpha_{i} \in H\left(X_{i}\right)$ maps to zero; the $f: t \rightarrow X_{i}$ were chosen so that the induced map $\operatorname{Hom}\left(t, X_{i}\right) \rightarrow H(t)$ vanishes. But $H$ is a homological functor; the exact sequence

$$
H\left(X_{i+1}\right) \stackrel{k}{\rightarrow} H\left(X_{i}\right) \stackrel{j}{\rightarrow} H\left(K_{i+1}\right),
$$

coupled with the fact that $j\left(\alpha_{i}\right)=0$, guarantees that there exists $\alpha_{i+1} \in H\left(X_{i+1}\right)$ with $k\left(\alpha_{i+1}\right)=\alpha_{i}$. Choose such an $\alpha_{i+1}$. There is a corresponding natural transformation

$$
\operatorname{Hom}\left(-, X_{i+1}\right) \rightarrow H
$$


rendering commutative the triangle:

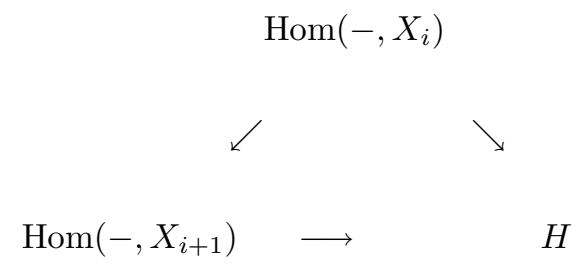

Let $X=\underset{\operatorname{hocolim}}{\longrightarrow} X_{i}$. I assert:

(1) There is a natural transformation $\operatorname{Hom}(-, X) \rightarrow H$ rendering commutative the triangles

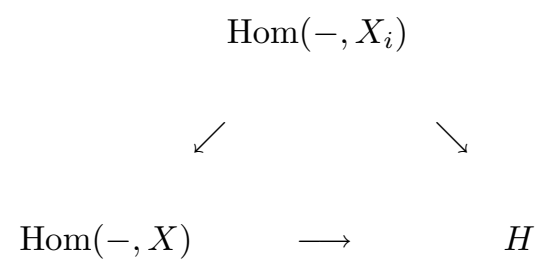

for every $i$.

(2) The natural transformation $\operatorname{Hom}(-, X) \rightarrow H$ is an isomorphism.

Proof of (1). Consider the triangle:

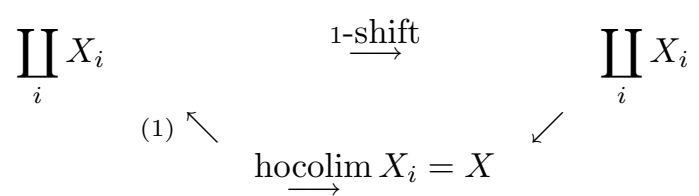

Applying the cohomological functor $H$, we get an exact sequence

$$
\begin{gathered}
H(X) \longrightarrow H\left(\coprod_{i} X_{i}\right) \stackrel{1-\text { shift }}{\longrightarrow} H\left(\coprod_{i} X_{i}\right) \\
\| \\
\prod_{i} H\left(X_{i}\right) \stackrel{1-\text { shift }}{\longrightarrow} \prod_{i} H\left(X_{i}\right) .
\end{gathered}
$$

The element

$$
\prod_{i} \alpha_{i} \in \prod_{i} H\left(X_{i}\right)
$$

is in the kernel of (1-shift), and hence there is an $\alpha \in H(X)$ mapping to it. By Yoneda, $\alpha$ corresponds to a natural transformation

$$
\operatorname{Hom}(-, X) \rightarrow H
$$


and the fact that $\alpha$ maps to $\prod \alpha_{i} \in H\left(\amalg X_{i}\right)$ means that the diagram

$$
\operatorname{Hom}\left(-, X_{i}\right)
$$

$$
\operatorname{Hom}(-, X) \quad \longrightarrow \quad H
$$

commutes for all $i$.

Proof of (2). It remains to show that

$$
\phi: \operatorname{Hom}(-, X) \rightarrow H
$$

constructed above is an isomorphism. Let us begin with objects $t \in T$. We will show that, for all $t \in T$,

$$
\phi(t): \operatorname{Hom}(t, X) \rightarrow H(t)
$$

is an isomorphism.

Observe the commutative diagram:

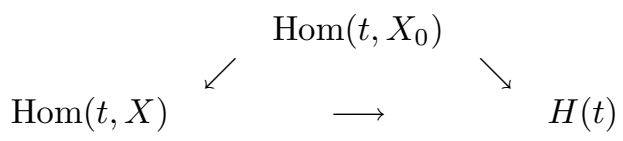

Since we know that $\operatorname{Hom}\left(t, X_{0}\right) \rightarrow H(t)$ is surjective, it follows that

$$
\operatorname{Hom}(t, X) \rightarrow H(t)
$$

is surjective. It remains only to prove it injective.

Let $f \in \operatorname{Hom}(t, X)$ with $\phi(t)(f)=0$. Now

$$
f \in \operatorname{Hom}(t, X)=\operatorname{Hom}\left(t, \underset{\operatorname{hocolim}}{\longrightarrow} X_{i}\right) .
$$

But as $t \in T$ is compact, we have by Lemma 2.8 that

$$
\operatorname{Hom}\left(t, \underset{\operatorname{hocolim}}{\longrightarrow} X_{i}\right)=\underset{\rightarrow}{\lim } \operatorname{Hom}\left(t, X_{i}\right) .
$$

In other words, there exists $f_{i}: t \rightarrow X_{i}$ so that the composite

$$
t \stackrel{f_{i}}{\longrightarrow} X_{i} \longrightarrow X
$$

is $f$. But the diagram

$$
\begin{array}{clll} 
& \operatorname{Hom}\left(t, X_{i}\right) & \\
\operatorname{Hom}(t, X) & & \searrow & \\
& & & H(t)
\end{array}
$$

commutes, and $j\left(f_{i}\right)=f$ while $k(f)=0$. It follows that $f_{i} \in \operatorname{ker}\left\{\operatorname{Hom}\left(t, X_{i}\right) \rightarrow\right.$ $H(t)\}$, that is, $\left(f_{i}, t\right) \in U_{i+1}$. This means that $f_{i}: t \rightarrow X_{i}$ factors through the map 
$h$ in the triangle

$$
\begin{gathered}
h \nearrow \\
\left\{\coprod_{\left(f_{i}, t\right) \in U_{i+1}} t\right\}=K_{i+1} \quad \longleftarrow g \\
\longleftarrow(1)
\end{gathered}
$$

and hence $g \circ f_{i}=0$. But the map

$$
X_{i} \stackrel{g}{\longrightarrow} X_{i+1} \stackrel{\bar{g}}{\longrightarrow} X
$$

satisfies

$$
\begin{aligned}
f & =\{\bar{g} \circ g\} \circ f_{i} \\
& =\bar{g} \circ\left\{g \circ f_{i}\right\} \\
& =0 .
\end{aligned}
$$

Thus $\phi(t): \operatorname{Hom}(t, X) \rightarrow H(t)$ is an isomorphism whenever $t \in T$.

Let $\mathcal{S} \subset \mathcal{T}$ be the full subcategory of objects $y \in \mathcal{T}$ such that, for all $n \in \mathbb{Z}$, the map $\phi\left(\Sigma^{n} y\right): \operatorname{Hom}\left(\Sigma^{n} y, X\right) \rightarrow H\left(\Sigma^{n} y\right)$ is an isomorphism. Then the category $\mathcal{S}$ contains $T$, and is closed with respect to the formation of coproducts and triangles. To finish our proof of Theorem 3.1, we need the following lemma.

Lemma 3.2. Let $\mathcal{S} \subset \mathcal{T}$ be a full, triangulated subcategory containing $T$ and closed under the formation of $\mathcal{T}$-coproducts of its objects. Then $\mathcal{S}=\mathcal{T}$.

Proof. Let $\mathcal{S}$ be the smallest subcategory of $\mathcal{T}$ which is full, triangulated, closed with respect to $\mathcal{T}$-coproducts of its objects, and contains $T$. Let $Z$ be an object of $\mathcal{T}$. Let $H=\operatorname{Hom}_{\mathcal{T}}(-, Z)$ be viewed as a homological functor on $\mathcal{S}$.

Then $\mathcal{S}$ is compactly generated, with a generating set $T$. We can therefore apply what we have proved so far about Brown representability to the functor $H$ on $\mathcal{S}$; there is an object $X$ of $\mathcal{S}$, a natural transformation of functors on $\mathcal{S}$

$$
\phi: \operatorname{Hom}_{\mathcal{S}}(-, X) \rightarrow \operatorname{Hom}_{\mathcal{T}}(-, Z),
$$

and this natural transformation is an isomorphism on a full, triangulated subcategory of $\mathcal{S}$ containing $T$ and closed with respect to $\mathcal{S}$-coproducts of its objects. But $\mathcal{S}$ is minimal with this property; hence

$$
\operatorname{Hom}_{\mathcal{S}}(-, X) \rightarrow \operatorname{Hom}_{\mathcal{T}}(-, Z)
$$

is an isomorphism of functors on $\mathcal{S}$.

By Yoneda's lemma, this means there is a morphism $X \rightarrow Z$ in $\mathcal{T}$ so that, for every object $s$ of $\mathcal{S}$,

$$
\operatorname{Hom}(s, X) \rightarrow \operatorname{Hom}(s, Z)
$$

is an isomorphism.

Complete $X \rightarrow Z$ to a triangle in $\mathcal{T}$ :

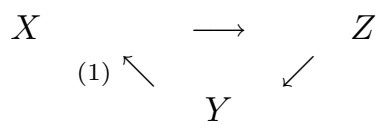


One easily deduces that, for every object $s$ of $\mathcal{S}, \operatorname{Hom}(s, Y)=0$. But $T \subset \mathcal{S}$, and hence for every object $t \in T$, $\operatorname{Hom}(t, Y)=0$. But because $T$ generates, $Y=0$, and hence $X \rightarrow Z$ is an isomorphism. Thus $Z$ is an object of $\mathcal{S}$, and since $Z \in \mathcal{T}$ was arbitrary, $\mathcal{S}=\mathcal{T}$.

\section{The ADJOINT FUNCTOR THEOREM AND EXAMPLES}

Theorem 4.1. Let $\mathcal{S}$ be a compactly generated triangulated category, $\mathcal{T}$ any triangulated category. Let $F: \mathcal{S} \rightarrow \mathcal{T}$ be a triangulated functor. Suppose $F$ respects coproducts; the natural maps

$$
F\left(s_{\lambda}\right) \rightarrow F\left(\coprod_{\lambda \in \Lambda} s_{\lambda}\right)
$$

make $F\left(\coprod_{\lambda \in \Lambda} s_{\lambda}\right)$ a coproduct of $\mathcal{T}$. Then $F$ has a right adjoint $G: \mathcal{T} \rightarrow \mathcal{S}$.

Proof. Let $t$ be an object of $\mathcal{T}$, and consider the functor on $\mathcal{S}$

$$
s \mapsto \operatorname{Hom}_{\mathcal{T}}(F(s), t) .
$$

This functor is homological and takes coproducts to products; we have

$$
\begin{aligned}
\operatorname{Hom}_{\mathcal{T}}\left(F\left(\coprod_{\lambda \in \Lambda} s_{\lambda}\right), t\right) & =\operatorname{Hom}_{\mathcal{T}}\left(\coprod_{\lambda \in \Lambda} F\left(s_{\lambda}\right), t\right) \\
& =\prod_{\lambda \in \Lambda} \operatorname{Hom}_{\mathcal{T}}\left(F\left(s_{\lambda}\right), t\right) .
\end{aligned}
$$

Hence, by Theorem 3.1, this functor is representable; there is a $G(t) \in \mathcal{S}$ with

$$
\operatorname{Hom}_{\mathcal{T}}(F(s), t)=\operatorname{Hom}_{\mathcal{S}}(s, G(t)),
$$

and by standard arguments, $G$ extends to a functor, right adjoint to $F$.

Example 4.2. Let $f: X \rightarrow Y$ be a separated morphism of quasi-compact separated schemes. Then

has a right adjoint

$$
R f_{*}: D(q c / X) \rightarrow D(q c / Y)
$$

$$
f^{!}: D(q c / Y) \rightarrow D(q c / X) .
$$

Proof. We need only show that $R f_{*}$ is triangulated and respects coproducts; the fact that it is triangulated is obvious, the fact that it respects coproducts is Lemma 1.4.

Example 4.3. Let $f: X \rightarrow Y$ be a separated morphism of smooth, quasi-compact, separated schemes of finite type over a field $k$ of characteristic 0 . Then

$$
R f_{+}: D\left(\frac{q c D \text {-modules }}{X}\right) \rightarrow D\left(\frac{q c D \text {-modules }}{Y}\right)
$$

has a left adjoint.

Proof. Since $R f_{+}$is clearly a triangulated functor, the real point is to prove that it respects coproducts. But $R f_{+}$is given as

$$
R f_{+}(x) \stackrel{\text { def }}{=} R f_{*}\left(\mathcal{D}_{Y \leftarrow X} \otimes_{\mathcal{D}_{X}} x\right)
$$

and tensor product trivially respects coproducts, while $R f_{*}$ does by Lemma 1.4. 
Example 4.4. With $f: X \rightarrow Y$ as in Example 4.3, let $D$ (holo/ $X$ ) and $D$ (holo/Y) be as in Example 2.7; that is, $D($ holo/ $X)$ is the smallest full, triangulated category of $D\left(\frac{q c D \text {-modules }}{X}\right)$ closed with respect to coproducts and containing the bounded complexes with holonomic cohomology. It is well known that

$$
R f_{+}: D\left(\frac{q c D \text {-modules }}{X}\right) \rightarrow D\left(\frac{q c D \text {-modules }}{Y}\right)
$$

takes complexes with holonomic cohomology to complexes with holonomic cohomology. Since $R f_{+}$is triangulated and respects coproducts, it takes $D($ holo $/ X$ ) to $D($ holo $/ Y)$. It induces a functor, which we also denote $R f_{+}$,

$$
R f_{+}: D(\text { holo } / X) \rightarrow D(\text { holo } / Y)
$$

This functor has a left adjoint.

Proof. By Example 2.7, $D$ (holo/ $X$ ) is a compactly generated triangulated category. The functor $R f_{+}$is the restriction to $D$ (holo/X) of a functor on $D\left(\frac{q c D \text {-modules }}{X}\right)$ respecting coproducts; hence it respects coproducts. By Theorem 3.1, the adjoint exists.

\section{Commuting with coproducts}

In Section 4, we constructed an adjoint to a functor $F: \mathcal{S} \rightarrow \mathcal{T}$. Precisely, if $F: \mathcal{S} \rightarrow \mathcal{T}$ is a triangulated functor, one sometimes has a right adjoint $G: \mathcal{T} \rightarrow \mathcal{S}$. Being a right adjoint, $G$ certainly respects products. It turns out to be interesting to know when $G$ respects coproducts.

Theorem 5.1. Let $\mathcal{S}$ be a compactly generated triangulated category, and let $\mathcal{T}$ be any triangulated category. Let $F: \mathcal{S} \rightarrow \mathcal{T}$ be a triangulated functor respecting coproducts, and let $G: \mathcal{T} \rightarrow \mathcal{S}$ be its right adjoint, which exists by Theorem 4.1. Let $S$ be a generating set for $\mathcal{S}$. Then $G: \mathcal{T} \rightarrow \mathcal{S}$ respects coproducts if and only if for every $s \in S, F(s)$ is a compact object of $\mathcal{T}$.

Proof. $\Rightarrow$ Suppose $G$ preserves coproducts. Let $s \in S$ be some object. Then

$$
\begin{array}{rlr}
\operatorname{Hom}_{\mathcal{T}}\left(F(s), \coprod_{\lambda \in \Lambda} x_{\lambda}\right) & =\operatorname{Hom}_{\mathcal{S}}\left(s, G\left(\coprod_{\lambda \in \Lambda} x_{\lambda}\right)\right) & \\
& =\operatorname{Hom}_{\mathcal{S}}\left(s, \coprod_{\lambda \in \Lambda} G\left(x_{\lambda}\right)\right) & \begin{array}{l}
\text { because } G \text { commutes } \\
\text { with coproducts }
\end{array} \\
& =\coprod_{\lambda \in \Lambda} \operatorname{Hom}_{\mathcal{S}}\left(s, G\left(x_{\lambda}\right)\right) & \text { because } s \text { is compact } \\
& =\coprod_{\lambda \in \Lambda} \operatorname{Hom}_{\mathcal{T}}\left(F(s), x_{\lambda}\right),
\end{array}
$$

and this proves that $F(s)$ is compact in $\mathcal{T}$. 
$\Leftarrow$ Suppose that, for all $s \in S, F(s)$ is compact. Let $\coprod_{\lambda \in \Lambda} x_{\lambda}$ be a coproduct in $\mathcal{T}$. Then for any $s \in S$,

$$
\begin{aligned}
& \operatorname{Hom}\left(s, G\left(\coprod_{\lambda \in \Lambda} x_{\lambda}\right)\right)=\operatorname{Hom}\left(F(s), \coprod_{\lambda \in \Lambda} x_{\lambda}\right) \\
& =\coprod_{\lambda \in \Lambda} \operatorname{Hom}\left(F(s), x_{\lambda}\right) \quad \text { because } F(s) \text { is compact } \\
& =\coprod_{\lambda \in \Lambda} \operatorname{Hom}\left(s, G\left(x_{\lambda}\right)\right) \\
& =\operatorname{Hom}\left(s, \coprod_{\lambda \in \Lambda} G\left(x_{\lambda}\right)\right) \quad \text { because } s \text { is compact. }
\end{aligned}
$$

In other words, the natural map

$$
\coprod_{\lambda \in \Lambda} G\left(x_{\lambda}\right) \rightarrow G\left(\coprod_{\lambda \in \Lambda} x_{\lambda}\right)
$$

induces a natural transformation

$$
\phi: \operatorname{Hom}_{\mathcal{S}}\left(-, \coprod_{\lambda \in \Lambda} G\left(x_{\lambda}\right)\right) \rightarrow \operatorname{Hom}_{\mathcal{S}}\left(-, G\left(\coprod_{\lambda \in \Lambda} x_{\lambda}\right)\right),
$$

and $\phi(s)$ is an isomorphism for all $s \in S$. But then in the triangle

$$
\coprod_{\lambda \in \Lambda} G\left(x_{\lambda}\right) \rightarrow G\left(\coprod_{\lambda \in \Lambda} x_{\lambda}\right) \rightarrow Z \rightarrow \Sigma\left\{\coprod_{\lambda \in \Lambda} G\left(x_{\lambda}\right)\right\}
$$

the object $Z$ must satisfy $\operatorname{Hom}(\mathcal{S}, Z)=0$. But as $\mathcal{S}$ generates, $Z=0$ and

$$
\coprod_{\lambda \in \Lambda} G\left(x_{\lambda}\right) \rightarrow G\left(\coprod_{\lambda \in \Lambda} x_{\lambda}\right)
$$

is an isomorphism.

Example 5.2. Let $f: X \rightarrow Y$ be a pseudo-coherent proper morphism of separated, quasi-compact schemes. Suppose that $f$ is of finite Tor-dimension. Then $R f_{*}: D(q c / X) \rightarrow D(q c / Y)$ takes perfect complexes to perfect complexes, by [9].

It follows that it takes a set of generators of $D(q c / X)$ to a set of compact objects of $D(q c / Y)$. Hence $f^{!}$commutes with coproducts. What makes this interesting is Theorem 5.4 which follows. But in the theorem we appeal to the projection formula, and since I do not know a reference which proves it in the generality we want, the following is the sketch of a proof.

Proposition 5.3. Let $f: X \rightarrow Y$ be a morphism of separated, quasi-compact schemes. Let $D(X)$ be the derived category of all $\mathcal{O}_{X}$-modules, $D(Y)$ the derived category of all $\mathcal{O}_{Y}$-modules. Let $y$ be an object of $D(Y), x$ an object of $D(X)$. Then there is a natural map, in $D(Y)$,

$$
y^{L} \otimes R f_{*} x \rightarrow R f_{*}\left\{L f^{*} y^{L} \otimes x\right\} .
$$

If $y$ is in $D(q c / Y) \subset D(Y)$ and $x$ is in $D(q c / X) \subset D(X)$, this natural map is an isomorphism. 
Proof. The existence and naturality of the map really comes from the definition of $R f_{*}, L f^{*}$ and the derived tensor product. To define $R f_{*}$ of an object in $D(X)$, one expresses the derived category $D(X)$ as a quotient of the the homotopy category $K(X)$ by the acyclic complexes $E(X)$, and notes that the subcategory $L(X)$ of Bousfield local objects in $K(X)$ with respect to $E(X)$ maps isomorphically to $D(X)$. Here, $L(X)$ consists of the special complexes of injectives of Spaltenstein's [15]. $R f_{*}$ is just $f_{*}$ on $L(X)$.

The tensor product, and $L f^{*}$, depend for their construction on the fact that $D(X)$ is also isomorphic to the subcategory of Bousfield colocal objects, denoted here $\tilde{L}(X)$. Concretely, these are complexes of objects $j ! \mathcal{O}_{U}$, where $j: U \rightarrow X$ is the inclusion of an open affine, and $j$ ! is extension by zero.

Replacing $y$ by a colocal object on $Y$ and $x$ by a local object on $X$, the tensor products become the natural ones and we have an isomorphism

$$
y \otimes f_{*} x \simeq f_{*}\left\{f^{*} y \otimes x\right\} .
$$

The left-hand side identifies immediately with $y^{L} \otimes R f_{*} x$, by definition of the derived tensor product and $R f_{*}$. On the right, the part in brackets identifies with $L f^{*} y^{L} \otimes x$, again by definition. Hence a natural isomorphism

$$
y^{L} \otimes R f_{*} x \simeq f_{*}\left\{L f^{*} y^{L} \otimes x\right\} .
$$

The problem is that, in general, $L f^{*} y^{L} \otimes x$ is not Bousfield local (=a complex of injectives), and hence $f_{*}$ of it is not the same as $R f_{*}$. But for any complex $x$, there is a natural map $f_{*} x \rightarrow R f_{*} x$, and this gives the natural map

$$
y^{L} \otimes R f_{*} x \rightarrow R f_{*}\left\{L f^{*} y^{L} \otimes x\right\} .
$$

It remains to show that the restriction of this map to the subcategories of complexes of quasi-coherents is an isomorphism.

Fix $x \in D(q c / X)$; we have a natural transformation of functors in $y \in D(q c / Y)$. First, the problem is now local in $Y$ and we may therefore assume $Y$ affine. Secondly, on the category $D(q c / X), R f_{*}$ respects coproducts by Lemma 1.4. Tensor product and $L f^{*}$ always respect sums, so the map is a natural transformation of two functors in $y$ respecting coproducts. For each $y \in D(q c / Y)$, let $\phi(y)$ be the map

$$
y^{L} \otimes R f_{*} x \rightarrow R f_{*}\left\{L f^{*} y^{L} \otimes x\right\} .
$$

Let $\mathcal{R} \subset D(q c / Y)$ be the full subcategory of all $y$ 's such that $\phi\left(\Sigma^{n} y\right)$ is an isomorphism for all $n$. The category $\mathcal{R}$ is closed with respect to triangles and coproducts. It clearly contains $\mathcal{O}_{Y}$. Since $Y$ is affine, $\mathcal{O}_{Y}$ is ample and generates $D(q c / Y)$ by Example 1.10. By Lemma 3.2, it follows that $\mathcal{R}$ is all of $D(q c / Y)$.

Theorem 5.4. Let $f: X \rightarrow Y$ be a morphism of schemes. Suppose $R f_{*}$ has a right adjoint $f^{!}$which commutes with coproducts. Suppose $Y$ is quasi-compact and separated. Then there is a natural isomorphism of functors $D(q c / Y) \rightarrow D(q c / X)$, which on objects gives

$$
f^{!}(y) \simeq\left(L f^{*} y\right) \otimes_{\mathcal{O}_{X}}\left(f^{!} \mathcal{O}_{Y}\right) .
$$

Conversely, if $f^{!}$is naturally isomorphic to the functor on the right, it respects coproducts.

Proof. $\Leftarrow$ Suppose we have a natural isomorphism of functors in $y$

$$
f^{!}(y) \simeq\left(L f^{*} y\right) \otimes_{\mathcal{O}_{Y}}\left(f^{!} \mathcal{O}_{Y}\right) .
$$


Since $L f^{*}$ has a right adjoint, it respects coproducts. Tensor products respect coproducts, so we deduce that $f^{!}$respects coproducts.

$\Rightarrow$ We will show that there is a natural map

$$
\left(L f^{*} y\right) \otimes_{\mathcal{O}_{X}}\left(f^{!} \mathcal{O}_{Y}\right) \rightarrow f^{!}(y)
$$

and that this map is an isomorphism whenever $y$ is compact. Then, if $f^{!}$respects coproducts, it will easily follow that this natural map is an isomorphism for all $y$.

Let us prove a slightly more general fact. We will actually show that, for any $y^{\prime}$ in $D(q c / Y)$, there is a natural map

$$
\left(L f^{*} y\right) \otimes_{\mathcal{O}_{X}}\left(f^{!} y^{\prime}\right) \rightarrow f^{!}\left(y \otimes_{\mathcal{O}_{Y}} y^{\prime}\right)
$$

which is an isomorphism if $y$ is compact. The case $y^{\prime}=\mathcal{O}_{Y}$ is then the above.

In any case, there is a natural map

$$
\mu: R f_{*} f^{!} y^{\prime} \rightarrow y^{\prime},
$$

the counit of adjunction. For every $y \in D(q c / Y)$,

$$
R f_{*}\left[\left(L f^{*} y\right) \otimes_{\mathcal{O}_{X}} f^{!} y^{\prime}\right]=y \otimes_{\mathcal{O}_{Y}} R f_{*} f^{!} y^{\prime}
$$

by the projection formula. Hence there is a natural map

$$
\begin{array}{r}
R f_{*}\left[L f^{*}(y) \otimes_{\mathcal{O}_{X}} f^{\prime} y^{\prime}\right]=y \otimes_{\mathcal{O}_{Y}} R f_{*} f^{!} y^{\prime} \\
\downarrow 1_{y} \otimes \mu \\
y \otimes_{\mathcal{O}_{Y}} y^{\prime} .
\end{array}
$$

By adjunction, we have a map

$$
L f^{*}(y) \otimes_{\mathcal{O}_{X}} f^{!} y^{\prime} \rightarrow f^{!}\left(y \otimes_{\mathcal{O}_{Y}} y^{\prime}\right) .
$$

This is our natural map. We need to show that for compact $y$ it is an isomorphism.

Let $y$ be compact, and let $x$ be an arbitrary complex in $D(q c / X)$. It suffices to show that the natural map above induces an isomorphism after applying $\operatorname{Hom}(x,-)$. Let us therefore reflect what $\operatorname{Hom}(x,-)$ does. To begin with, put $\hat{y}=\mathcal{R} \mathcal{H} \operatorname{om}_{\mathcal{O}_{Y}}\left(y, \mathcal{O}_{Y}\right)$. Then $L f^{*} \hat{y}=\mathcal{R} \mathcal{H} o m_{\mathcal{O}_{X}}\left(L f^{*} y, \mathcal{O}_{X}\right)$, and since $y$ and $L f^{*} y$ are perfect complexes, there are natural isomorphisms

$$
\operatorname{Hom}_{X}\left(-\otimes L f^{*} \hat{y},-\right)=\operatorname{Hom}_{X}\left(-, L f^{*} y \otimes-\right)
$$

and

Now, the map

$$
\operatorname{Hom}_{Y}(-\otimes \hat{y},-)=\operatorname{Hom}_{Y}(-, y \otimes-)
$$

$$
L f^{*}(y) \otimes_{\mathcal{O}_{X}} f^{!} y^{\prime} \rightarrow f^{!}\left(y \otimes_{\mathcal{O}_{Y}} y^{\prime}\right)
$$

induces a map after applying $\operatorname{Hom}(x,-)$. By definition, this takes a map $\gamma \in \operatorname{Hom}\left(x, L f^{*}(y) \otimes_{\mathcal{O}_{X}} f^{!} y^{\prime}\right)$ to a map $x \rightarrow f^{!}\left(y \otimes_{\mathcal{O}_{Y}} y^{\prime}\right)$. By the adjunction, this map corresponds to a map $\gamma^{\prime}: R f_{*} x \rightarrow y \otimes_{\mathcal{O}_{Y}} y^{\prime}$. But we know what $\gamma^{\prime}$ is; it is the composite of $R f_{*} \gamma$ with the natural counit

$$
\begin{array}{r}
R f_{*}\left[L f^{*}(y) \otimes \mathcal{O}_{X} f^{!} y^{\prime}\right]=y \otimes_{\mathcal{O}_{Y}} R f_{*} f^{!} y^{\prime} \\
\downarrow 1_{y} \otimes \mu \\
y \otimes_{\mathcal{O}_{Y}} y^{\prime} .
\end{array}
$$


We need to show this correspondence is an isomorphism. But

$$
\begin{aligned}
\operatorname{Hom}_{\mathcal{O}_{X}}\left(x, L f^{*} y \otimes_{\mathcal{O}_{X}} f^{!} y^{\prime}\right) & =\operatorname{Hom}_{\mathcal{O}_{X}}\left(x \otimes L f^{*} \hat{y}, f^{!} y^{\prime}\right) \\
& =\operatorname{Hom}_{\mathcal{O}_{Y}}\left(R f_{*}\left\{x \otimes L f^{*} \hat{y}\right\}, y^{\prime}\right) \\
& =\operatorname{Hom}_{\mathcal{O}_{Y}}\left(R f_{*}(x) \otimes \hat{y}, y^{\prime}\right) \\
& =\operatorname{Hom}_{\mathcal{O}_{Y}}\left(R f_{*}(x), y \otimes \mathcal{O}_{Y} y^{\prime}\right),
\end{aligned}
$$

where the third equality is the projection formula. This isomorphism is easily identified with $\operatorname{Hom}(x,-)$ applied to the map

$$
\phi: L f^{*}(y) \otimes_{\mathcal{O}_{X}} f^{!} y^{\prime} \rightarrow f^{!}\left(y \otimes_{\mathcal{O}_{Y}} y^{\prime}\right) .
$$

We therefore know that $\phi$ is an isomorphism if $y$ is compact.

Now assume that $f^{!}$respects coproducts. For fixed $y^{\prime}$, the functor

$$
y \mapsto\left(L f^{*} y\right) \otimes_{\mathcal{O}_{X}} f^{!} y^{\prime}
$$

is a triangulated functor in $y ; L f^{*}$ is, as is tensor product. The functor $f^{!}$is the adjoint of a triangulated functor, hence triangulated; see [12], Lemma 3.9. Thus $\phi$ is a natural transformation of triangulated functors, both of which respect coproducts. Let $\mathcal{S} \subset D(q c / Y)$ be the full subcategory

$$
\mathcal{S}=\left\{y \in \mathcal{O} b[D(q c / Y)] \mid \phi\left(\Sigma^{n} y\right) \text { is an isomorphism for all } n \in \mathbb{Z}\right\} .
$$

Then $\mathcal{S}$ contains the generating set of compact objects, is triangulated and closed with respect to $D(q c / Y)$-coproducts. Thus $\mathcal{S}=D(q c / Y)$. Hence, $\phi$ is a natural isomorphism. The theorem is the special case $y^{\prime}=\mathcal{O}_{Y}$ of the above.

Remark 5.5. In the traditional literature on the subject, $f^{!} \mathcal{O}_{Y}$ is called the dualizing complex, and plays a key role in the theory.

\section{A SHEAF VERSION}

The traditional way to state Grothendieck's duality theorem comes in a sheaf version. Let $f: X \rightarrow Y$ be a proper morphism of noetherian, separated schemes. One would like to deduce that $f^{!}$, the adjoint we have for $R f_{*}$, gives an isomorphism in the category of sheaf homomorphisms

$$
\mathcal{R H o m}\left(R f_{*} x, y\right) \simeq R f_{*} \mathcal{R H o m}\left(x, f^{!} y\right) .
$$

Note that the counit $u: R f_{*} f^{!} y \rightarrow y$ defines in any case a well-defined natural transformation

$$
\phi: R f_{*} \mathcal{R H o m}\left(x, f^{!} y\right) \rightarrow \mathcal{R H o m}\left(R f_{*} x, y\right),
$$

and the only question is whether it is an isomorphism. Once we apply the functor $H^{0}(Y,-)$, it becomes an isomorphism; this is because $f^{!}$is adjoint to $R f_{*}$. To say that the map is an isomorphism of sheaves is to say that the derived functor of $\Gamma(U,-)$ gives an isomorphism $R \Gamma(U, \phi)$ for every open set $U \subset Y$. Concretely, it says that if we take the commutative diagram

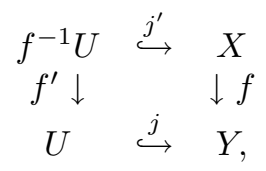

then $\left(f^{\prime}\right) ! j^{*}$ and $\left(j^{\prime}\right)^{*} f^{!}$are naturally isomorphic.

It is easy to see that $j^{*} R f_{*}=R f_{*}^{\prime}\left(j^{\prime}\right)^{*}$. Taking right adjoints, we deduce 
Lemma 6.1. If the diagram

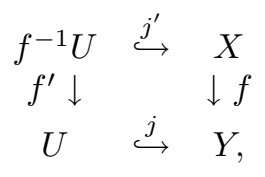

is given by pulling back an open immersion $j: U \rightarrow Y$, then there is a natural isomorphism

$$
f^{!} R j_{*}=R j_{*}^{\prime}\left(f^{\prime}\right)^{!} .
$$

Therefore

$$
\left(j^{\prime}\right)^{*}\left\{R j_{*}^{\prime}\left(f^{\prime}\right)^{!}\right\} j^{*}=\left(j^{\prime}\right)^{*}\left\{f^{!} R j_{*}\right\} j^{*} .
$$

Hence $\left(f^{\prime}\right)^{!} j^{*}=\left(j^{\prime}\right)^{*} f^{!} R j_{*} j^{*}$ because $\left(j^{\prime}\right)^{*} R j_{*}^{\prime}$ is the identity functor. Thus we must convince ourselves that the natural unit of adjunction $1 \rightarrow R j_{*} j^{*}$ induces an isomorphism

$$
\left(j^{\prime}\right)^{*} f^{!} \rightarrow\left(j^{\prime}\right)^{*} f^{!} R j_{*} j^{*} .
$$

Let $Z=Y-U$ be the (closed) complement of $U \subset Y$. There is a triangle of functors

$$
R \Gamma_{Z} \rightarrow 1 \rightarrow R j_{*} j^{*}
$$

where $R \Gamma_{Z}$ is Grothendieck's local cohomology functor. Sometimes $R \Gamma_{Z}$ is denoted $i_{*} i^{!}$, because it is also the counit of an adjunction. But since the $i^{!}$and $i_{*}$ are not the type of map we have been considering here, the notation might lead to confusion. Note that $Z \subset Y$ is a Zariski closed subset, but we have not given it a scheme structure. Hence the $i_{*}$ and $i^{!}$of this article make no sense.

It suffices to show that

$$
\left(j^{\prime}\right)^{*} f^{!} R \Gamma_{Z}
$$

is the zero functor. It is enough to show this for open sets $U \subset X$ which form a basis for the topology. This is what we will do.

Proposition 6.2. Let $f: X \rightarrow Y$ be a morphism of schemes such that $R f_{*}$ : $D(q c / X) \rightarrow D(q c / Y)$ has a right adjoint $f^{!}$. Suppose $f^{!}$respects coproducts. Suppose $U \subset Y$ is an open subset, $Z=Y-U$ the complement. Suppose $U$ and $Y$ are quasi-compact and separated. Then, in the notation above, the composite

$$
\left(j^{\prime}\right)^{*} f^{!} R \Gamma_{Z}=0 .
$$

Proof. We wish to show that $\left(j^{\prime}\right)^{*} f^{!}$vanishes on any object of the form $R \Gamma_{Z}(y)$. This means concretely that if $y$ is a complex which is acyclic away from $Z$, then $f^{!} y$ must be shown acyclic off $f^{-1} Z$. But by Theorem 5.4,

$$
f^{!} y=L f^{*} y \otimes f^{!} \mathcal{O}_{Y} .
$$

Clearly, if $y$ is supported on $Z$, then $L f^{*} y$ is supported on $f^{-1} Z$, and hence so is its tensor product with $f^{!} \mathcal{O}_{Y}$.

From the work of Verdier for the Noetherian case, Lipman in general, we know that for the bounded-below derived category more is true. Let us state their theorem, then prove it by coproduct techniques. 
Proposition 6.3. Let $f: X \rightarrow Y$ be a pseudo-coherent, proper morphism of quasicompact, separated schemes. Then $R f_{*}: D^{+}(q c / X) \rightarrow D^{+}(q c / Y)$ has a right adjoint $f^{!}$. Furthermore, in the notation above, the composite

$$
\left(j^{\prime}\right)^{*} f^{!} R \Gamma_{Z}=0 .
$$

Proof. Let us begin by showing that the right adjoint of $f^{!}: D(q c / Y) \rightarrow D(q c / X)$ takes bounded-below complexes to bounded-below complexes, and is therefore also a right adjoint to $R f_{*}: D^{+}(q c / X) \rightarrow D^{+}(q c / Y)$. Since $X$ is quasi-compact, it may be covered by finitely many open affines, say $n$ of them. But since $f$ is separated, the open affines may be used to compute $R f_{*}$, via the Cech complex. Since the Čech complex has only $(n+1)$ terms, it follows that if $x \in D^{-}(q c / X)$ vanishes above dimension $l$, then $R f_{*} x$ vanishes above dimension $l+n$. In the notation of $t$-structure truncations, if $x \in D(q c / X)^{\leq l}$, then $R f_{*} x \in D(q c / Y)^{\leq l+n}$.

Pick any $x \in D(q c / X)^{\leq l}$, and $y \in D(q c / Y)^{\geq l+n+1}$. Then

$$
\operatorname{Hom}\left(x, f^{!} y\right)=\operatorname{Hom}\left(R f_{*} x, y\right)=0
$$

since $R f_{*} x \in D(q c / Y)^{\leq l+n}$ and $y \in D(q c / Y)^{\geq l+n+1}$. But $x \in D(q c / X)^{\leq l}$ was arbitrary; thus $f^{!} y \in D(q c / X)^{\geq l+1}$. In other words, $y \in D(q c / Y)^{\geq l}$ implies $f^{!} y \in$ $D(q c / X)^{\geq l-n}$. In particular, if $y \in D^{+}(q c / Y)$, then $f^{!} y \in D^{+}(q c / X)$.

It remains to show the vanishing of $\left(j^{\prime}\right)^{*} f^{!} R \Gamma_{Z}$. We need to show that if $y$ is an object of $D^{+}(q c / Y)$, which is acyclic off $Z$, then $f^{!} y$ is acyclic off $f^{-1} Z$. Let us first make an observation. Suppose $y$ is arbitrary, vanishing off $Z$. Suppose $Y=V_{1} \cup V_{2}$ expresses $Y$ as a union of two open sets $V_{1}$ and $V_{2}$. There is then a triangle

$$
y \rightarrow j_{V_{1 *}} j_{V_{1}}^{*} y \oplus j_{V_{2} *} j_{V_{2}}^{*} y \rightarrow j_{V_{1} \cap V_{2} *} j_{V_{1} \cap V_{2}} * y \longrightarrow \Sigma y
$$

where $j_{W}: W \hookrightarrow Y$ is the inclusion of the open set $W$ in $Y$. From the triangle, it clearly suffices to show the vanishing of $\left(j^{\prime}\right)^{*} f^{!}$on the complexes $j_{W *} j_{W}{ }^{*} y$ where $W$ is any of $V_{1}, V_{2}$ or $V_{1} \cap V_{2}$. Suppose $X$ can be covered by $n$ affines. If $V_{1}$ is affine and $V_{2}$ the union of $n-1$ affines, then each of $V_{1}, V_{2}$ and $V_{1} \cap V_{2}$ can be covered by at most $n-1$ affines. By induction we therefore easily show that it suffices to prove the vanishing of $\left(j^{\prime}\right)^{*} f^{!}$on $j_{W *} j_{W}{ }^{*} y$ where $W \subset X$ is affine; in other words, we need to show that $f^{!} j_{W *} j_{W}{ }^{*} y$ vanishes off $f^{-1} Z$.

Even better, we may replace $y$ by $z=j_{W}{ }^{*} y$. It will suffice to show that, given an open affine $W \subset Y$ and $z \in D^{+}(q c / W)$ whose support is in $Z \cap W$, then $f^{!} j_{W *} z$ is supported on $f^{-1} Z$. Now consider the pullback square

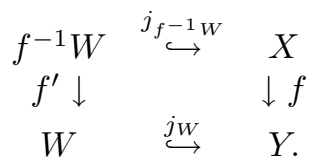

By Lemma 6.1 there is a natural isomorphism $\left\{j_{f^{-1} W}\right\}_{*}\left\{f^{\prime}\right\}^{!} z=f^{!}\left\{j_{W}\right\}_{*} z$. We need to show that this complex (either of the two isomorphic versions) is acyclic off $f^{-1} Z$. From the description as $\left\{j_{f^{-1} W}\right\}_{*}\left\{f^{\prime}\right\}^{!} z$, it clearly suffices to show that $\left\{f^{\prime}\right\}^{!} z$ is supported on $f^{-1}\{Z \cap W\}$. In other words, we are reduced to studying the problem for the map $f: f^{-1} W \rightarrow W$. Thus we may assume $Y$ affine.

Next it is clear that if $Z=\bigcap Z_{i}$, it is enough to prove the statement for each $Z_{i}$. We may therefore assume that $Z$ is a "divisor" in $W$. That is, there exists a global function $\gamma \in \Gamma\left(Y, \mathcal{O}_{Y}\right)$, so that $Z$ is the divisor defined by $\gamma$. 
On $Y$ we have perfect complexes, namely the desuspensions of the mapping cones of the maps

$$
\gamma^{k}: \mathcal{O}_{Y} \rightarrow \mathcal{O}_{Y}
$$

Call these perfect complexes $b_{k}$. Then $b_{k}$ fits in a triangle

$$
b_{k} \rightarrow \mathcal{O}_{Y} \stackrel{\gamma^{k}}{\rightarrow} \mathcal{O}_{Y} \rightarrow \Sigma b_{k} .
$$

There is a map $b_{k} \rightarrow b_{k+1}$, given by completing the commutative square

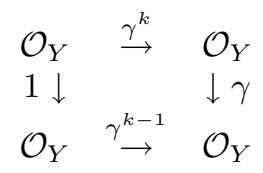

to a map of triangles

$$
\begin{array}{ccccccc}
b_{k} & \rightarrow & \mathcal{O}_{Y} & \stackrel{\gamma^{k}}{\rightarrow} & \mathcal{O}_{Y} & \rightarrow & \Sigma b_{k} \\
\downarrow & 1 \downarrow & & \downarrow \gamma & & \downarrow \\
b_{k+1} & \rightarrow & \mathcal{O}_{Y} & \stackrel{\gamma^{k+1}}{\rightarrow} & \mathcal{O}_{Y} & \rightarrow & \Sigma b_{k+1}
\end{array}
$$

There is furthermore a map $b_{k} \rightarrow \mathcal{O}_{Y}$, which is a map from the direct system.

Applying the functor $(-) \otimes z$ to this direct system, we get a direct system with a map

$$
b_{k} \otimes z \rightarrow \mathcal{O}_{Y} \otimes z=z .
$$

This induces a (non-canonical) map on homotopy colimits

$$
\operatorname{hocolim}\left(b_{k} \otimes z\right) \rightarrow z
$$

and since the cohomology of $z$ is supported on $Z$, that is annihilated by some power of $\gamma$, it is easy to show that the map is a cohomology isomorphism, hence an isomorphism in the derived category.

Note that in the construction above, $b_{k} \otimes z$ can be obtained by tensoring the triangle

$$
b_{k} \rightarrow \mathcal{O}_{Y} \stackrel{\gamma^{k}}{\rightarrow} \mathcal{O}_{Y} \rightarrow \Sigma b_{k}
$$

with the object $z$. There is a triangle

$$
b_{k} \otimes z \rightarrow z \stackrel{\gamma^{k}}{\rightarrow} z \rightarrow \Sigma b_{k} \otimes z
$$

We know that $z \in D^{+}(q c / Y)$. Suppose $z \in D(q c / Y)^{\geq l}$. Then from the triangle, $b_{k} \otimes z$ also lies in $D(q c / Y)^{\geq l}$.

Because $z$ is the homotopy colimit of $b_{k} \otimes z$, there is a triangle on $Y$

$$
\bigoplus_{k}\left[b_{k} \otimes z\right] \rightarrow \bigoplus_{k}\left[b_{k} \otimes z\right] \rightarrow z \rightarrow \Sigma \bigoplus_{k}\left[b_{k} \otimes z\right]
$$

which expresses $z$ as the homotopy colimit. Applying $f^{!}$, we have a triangle

$$
f^{!}\left\{\bigoplus_{k}\left[b_{k} \otimes z\right]\right\} \rightarrow f^{!}\left\{\bigoplus_{k}\left[b_{k} \otimes z\right]\right\} \rightarrow f^{!} z \rightarrow f^{!}\left\{\Sigma \bigoplus_{k}\left[b_{k} \otimes z\right]\right\}
$$

and to show that $f^{!} z$ is supported on $f^{-1} Z$, it suffices to show that the other two terms in the triangle are. Now note that in any case there is a natural isomorphism

$$
f^{!}\left[b_{k} \otimes z\right]=L f^{*} b_{k} \otimes f^{!} z
$$


because $b_{k}$ is compact, and by the proof of Theorem 5.4. Now $L f^{*} b_{k}$ is supported on $f^{-1} Z$, hence its tensor product with $f^{!} j_{W *} z$ also is. It follows that $f^{!}\left[b_{k} \otimes z\right]$ is supported on $f^{-1} Z$. We need to show that $f^{!}\left\{\bigoplus_{k}\left[b_{k} \otimes z\right]\right\}$ is supported on $f^{-1} Z$. It will clearly suffice to show that the natural map

$$
\bigoplus_{k} f^{!}\left[b_{k} \otimes z\right] \rightarrow f^{!}\left\{\bigoplus_{k}\left[b_{k} \otimes z\right]\right\}
$$

is an isomorphism. This we will now do.

Now let $x$ be an arbitrary perfect complex on $X$. Since we are only assuming that the map $f: X \rightarrow Y$ is proper and of finite type, we do not know that $R f_{*} x$ is perfect. However, we do know, by [9], that locally it can be resolved by finite dimensional vector bundles to arbitrary length. That is, $Y$ can be covered by open sets $V$, and for each $V$ there is a triangle

$$
q \rightarrow p \rightarrow R f_{*} x \longrightarrow \Sigma q
$$

where $p$ is perfect and $q \in D(q c / V)^{\leq l-1}$. Since $Y$ is affine, this can even be done globally. Such a triangle exists on all of $Y$. We deduce

$$
\begin{aligned}
\operatorname{Hom}\left(x, f^{!}\left\{\bigoplus_{k}\left[b_{k} \otimes z\right]\right\}\right) & =\operatorname{Hom}\left(R f_{*} x, \bigoplus_{k}\left[b_{k} \otimes z\right]\right) \\
& =\operatorname{Hom}\left(p, \bigoplus_{k}\left[b_{k} \otimes z\right]\right) \\
& =\bigoplus_{k} \operatorname{Hom}\left(p,\left[b_{k} \otimes z\right]\right) \\
& =\bigoplus_{k} \operatorname{Hom}\left(R f_{*} x,\left[b_{k} \otimes z\right]\right) \\
& =\bigoplus_{k} \operatorname{Hom}\left(x, f^{!}\left[b_{k} \otimes z\right]\right) \\
& =\operatorname{Hom}\left(x, \bigoplus_{k} f^{!}\left[b_{k} \otimes z\right]\right) .
\end{aligned}
$$

But we have a map, defined on $Y$,

$$
\bigoplus_{k} f^{!}\left[b_{k} \otimes z\right] \rightarrow f^{!}\left\{\bigoplus_{k}\left[b_{k} \otimes z\right]\right\}
$$

and we have just shown that if we apply the functor $\operatorname{Hom}(x,-)$ to this map where $x \in D(q c / X)$ is compact, we get an isomorphism. But then the map

$$
\operatorname{Hom}\left(x, \bigoplus_{k} f^{!}\left[b_{k} \otimes z\right]\right) \rightarrow \operatorname{Hom}\left(x, f^{!}\left\{\bigoplus_{k}\left[b_{k} \otimes z\right]\right\}\right)
$$


is an isomorphism for all $x$ in the subcategory generated by the compacts in $D(q c / X)$; that is, for any $x \in D(q c / X)$. Thus the map

$$
\bigoplus_{k} f^{!}\left[b_{k} \otimes z\right] \rightarrow f^{!}\left\{\bigoplus_{k}\left[b_{k} \otimes z\right]\right\}
$$

is an isomorphism in $D(q c / X)$.

Remark 6.4. The results of Verdier apply not only to open immersions, but to arbitrary flat maps. In other words, he proves, in Theorem 2 of [17], the following. Suppose we have a cartesian square of noetherian schemes

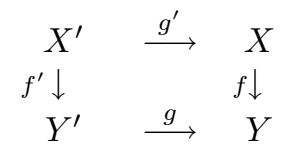

with $f$ and $f^{\prime}$ proper, $g$ and $g^{\prime}$ flat. Then there is a natural isomorphism

$$
\left\{g^{\prime}\right\}^{*} f^{!}=\left\{f^{\prime}\right\}^{!} g^{*}
$$

What interests us here is not so much the best statement possible, but the relation with preserving coproducts. To illustrate this, we will give the following counterexample.

Example 6.5. Let $R$ be a noetherian ring (e.g. $\mathbb{Z}$ ), and let $S=R[\epsilon] /\left(\epsilon^{2}\right)$. There is a homomorphism $S \rightarrow R$ sending $\epsilon$ to 0 . This gives a map of schemes from $X=\operatorname{Spec}(R)$ to $Y=\operatorname{Spec}(S)$. This map is certainly proper, and of finite type. Let us denote this map $f: X \rightarrow Y$.

For affine maps, $f^{!}$is easy to describe. For any $S$-module $N$ and $R$-module $M$, we can view $M$ as an $S$-module via the homomorphism $S \rightarrow R$. This is the functor

$$
f_{*}:\{R-\bmod \} \rightarrow\{S-\bmod \} .
$$

There is a canonical isomorphism

$$
\operatorname{Hom}_{S}(M, N)=\operatorname{Hom}_{R}\left(M, \operatorname{Hom}_{S}(R, N)\right) .
$$

This allows us to view $f^{!}$as the derived Hom,

$$
f^{!} N=R \operatorname{Hom}_{S}(R, N),
$$

for any $N \in D(q c / Y)=D(S)$. One way to get the derived functor of Hom is to take projective resolutions in the first variable. There is an obvious projective resolution for $R$ as an $S$-module; the chain complex

$$
\cdots \stackrel{\epsilon}{\rightarrow} S \stackrel{\epsilon}{\rightarrow} S \rightarrow R \rightarrow 0
$$

is exact. Let $N$ be $R$, viewed as an $S$-module. Then

$$
f^{!} R=R \operatorname{Hom}_{S}(R, R)=\prod_{i=0}^{\infty} \Sigma^{-i} R
$$

is the complex which is $R$ in every positive dimension, with differential zero. 
Now consider the complex

$$
N=\prod_{k=0}^{\infty} \Sigma^{k} R
$$

that is, the complex with zero differential which is $R$ in every negative dimension, viewed as a complex of $S$-modules. Because $f^{!}$is a right adjoint, it respects products. Thus,

$$
\begin{aligned}
f^{!} N & =f^{!}\left\{\prod_{k=0}^{\infty} \Sigma^{k} R\right\} \\
& =\prod_{k=0}^{\infty} f^{!}\left\{\Sigma^{k} R\right\} \\
& =\prod_{k=0}^{\infty} \prod_{i=0}^{\infty} \Sigma^{k-i} R .
\end{aligned}
$$

Choose an element $\gamma \in R \subset S=R[\epsilon] /\left(\epsilon^{2}\right)$. If we restrict to the open subset where $\gamma$ is inverted, then $f^{!} N$ restricts to

$$
\left\{j^{\prime}\right\}^{*} f^{!} N=\left\{f^{!} N\right\} \otimes_{R} R\left[\frac{1}{\gamma}\right] .
$$

On the other hand, if we first restrict to the open subset, note that we are in exactly the same situation; $S\left[\frac{1}{\gamma}\right]=R\left[\frac{1}{\gamma}\right][\epsilon] /\left(\epsilon^{2}\right)$. The complex $N$ restricts to

$$
j^{*} N=\prod_{k=0}^{\infty} \Sigma^{k} R\left[\frac{1}{\gamma}\right] .
$$

If this is not clear, note that because the $R$ 's are placed in different dimensions, the coproduct agrees with the product. The natural map

$$
\bigoplus_{k=0}^{\infty} \Sigma^{k} R \rightarrow \prod_{k=0}^{\infty} \Sigma^{k} R=N
$$

is a homology isomorphism. But $j^{*}$, being a left adjoint, respects coproducts; hence

$$
j^{*}\left(\bigoplus_{k=0}^{\infty} \Sigma^{k} R\right)=\bigoplus_{k=0}^{\infty} j^{*} \Sigma^{k} R=\bigoplus_{k=0}^{\infty} \Sigma^{k} R\left[\frac{1}{\gamma}\right]
$$

and, once again, the natural map

$$
\bigoplus_{k=0}^{\infty} \Sigma^{k} R\left[\frac{1}{\gamma}\right] \rightarrow \prod_{k=0}^{\infty} \Sigma^{k} R\left[\frac{1}{\gamma}\right]
$$

is a homology isomorphism. We can therefore use our last computation, replacing $R$ by $R\left[\frac{1}{\gamma}\right]$, to deduce that

$$
\left\{f^{\prime}\right\}^{!} j^{*} N=\prod_{k=0}^{\infty} \prod_{i=0}^{\infty} \Sigma^{k-i} R\left[\frac{1}{\gamma}\right] .
$$


In other words, we have just computed everything. It remains to check whether the natural map is an isomorphism $\left\{j^{\prime}\right\}^{*} f^{!} N \rightarrow\left\{f^{\prime}\right\}^{!} j^{*} N$. The map is just

$$
\left\{\prod_{k=0}^{\infty} \prod_{i=0}^{\infty} \Sigma^{k-i} R\right\} \otimes_{R} R\left[\frac{1}{\gamma}\right] \rightarrow \prod_{k=0}^{\infty} \prod_{i=0}^{\infty} \Sigma^{k-i} R\left[\frac{1}{\gamma}\right] .
$$

If we just look at the induced map on $H^{0}$, the product is over all $k=i$. The map on $H^{0}$ is therefore

$$
\left\{\prod_{i=0}^{\infty} R\right\} \otimes_{R} R\left[\frac{1}{\gamma}\right] \rightarrow \prod_{i=0}^{\infty} R\left[\frac{1}{\gamma}\right] .
$$

This map clearly is not an isomorphism in general. If $\gamma$ is neither nilpotent nor invertible in $R$, then the element

$$
\prod_{i=0}^{\infty} \frac{1}{\gamma^{i}}
$$

is a well-defined member of the right-hand side, but not the image of something on the left.

\section{REFERENCES}

[1] P. Berthelot, A. Grothendieck and L. Illusie, SGA 6: Théorie des intersections et théorème de Riemann-Roch, Lecture Notes in Math., vol. 225, Springer-Verlag, Heidelberg, 1971. MR 50:7133

[2] M. Bökstedt and A. Neeman, Homotopy limits in triangulated categories, Compositio Math. 86 (1993), 209-234. MR 94f: 18008

[3] A.K. Bousfield, The localization of spaces with respect to homology, Topology 14 (1975), 133-150. MR 52:1676

[4] , The localization of spectra with respect to homology, Topology 18 (1979), 257-281. MR 80m:55006

[5] _ The Boolean algebra of spectra, Comm. Math. Helv. 54 (1979), 368-377. MR 81a:55015

[6] E.H. Brown, Abstract homotopy theory, Trans. Amer. Math. Soc. 119 (1965), 79-85. MR 32:452

[7] P. Deligne, Cohomology à support propre en construction du foncteur $f^{!}$, Appendix to: Residues and Duality, Lecture Notes in Math., vol. 20, Springer-Verlag, Heidelberg, 1966, pp. 404-421. MR 36:5145

[8] R. Hartshorne, Residues and duality, Lecture Notes in Math., vol. 20, Springer-Verlag, Heidelberg, 1966. MR 36:5145

[9] R. Kiehl, Ein "Descente"-Lemma und Grothendiecks Projektionssatz für nichtnoethersche Schemata, Math Ann. 198 (1972), 287-316. MR 52:3165

[10] J. Lipman, Notes on derived categories and derived functors, preprint.

[11] _ Non-noetherian Grothendieck duality, preprint.

[12] A. Neeman, Stable homotopy as a triangulated functor, Invent. Math. 109 (1992), 17-40. MR 93j:55022

[13] _ The connection between the K-theory localisation theorem of Thomason, Trobaugh and Yao and the smashing subcategories of Bousfield and Ravenel, Ann. Sci. École Norm. Sup. 25 (1992), 547-566. MR 93k:18015

[14] A. Neeman and V.Voevodsky, Triangulated categories, incomplete preprint.

[15] N. Spaltenstein, Resolutions of unbounded complexes, Compositio Math. 65 (1988), 121-154. MR 89m:18013 
[16] R. Thomason and T. Trobaugh, Higher algebraic K-theory of schemes and of derived categories, The Grothendieck Festschrift ( a collection of papers to honor Grothendieck's 60'th birthday), Vol. 3, Birkhäuser, 1990, pp. 247-435. MR 92f:19001

[17] J.-L. Verdier, Base change for twisted inverse images of coherent sheaves, Collection: Algebraic Geometry (Internat. Colloq.), Tata Inst. Fund. Res., Bombay, 1968, pp. 393-408. MR 43:227

ABstract. Grothendieck proved that if $f: X \longrightarrow Y$ is a proper morphism of nice schemes, then $R f_{*}$ has a right adjoint, which is given as tensor product with the relative canonical bundle. The original proof was by patching local data. Deligne proved the existence of the adjoint by a global argument, and Verdier showed that this global adjoint may be computed locally.

In this article we show that the existence of the adjoint is an immediate consequence of Brown's representability theorem. 1It follows almost as immediately, by "smashing" arguments, that the adjoint is given by tensor product with a dualising complex. Verdier's base change theorem is an easy consequence.

Department of Mathematics, University of Virginia, Charlottesville, Virginia 22903

E-mail address: an3r@virginia.edu 